A DYNAMIC MODEL OF THE MAGNETIC HEAD SLIDER WITH CONTACT AND OFF-TRACK MOTION DUE TO A THERMALLY ACTUATED PROTRUSION OR A MOVING BUMP INVOLVING INTERMOLECULAR FORCES

\author{
A Thesis \\ Presented to \\ The Graduate Faculty of The University of Akron
}

In Partial Fulfillment

Of the Requirements for the Degree

Master of Science

Saurabh Pathak

August, 2016 


\section{A DYNAMIC MODEL OF THE MAGNETIC HEAD SLIDER WITH CONTACT AND OFF-TRACK MOTION DUE TO A THERMALLY ACTUATED PROTRUSION OR A MOVING BUMP INVOLVING INTERMOLECULAR FORCES}

Saurabh Pathak

Thesis

Approved:

Co-Advisor

Dr. Shao Wang

Co-Advisor

Dr. Shing-Chung “Josh” Wong

Faculty Reader

Dr. Graham Kelly
Accepted:

Interim Dean of the College

Dr. Eric J. Amis

Dean of the Graduate School

Dr. Chand K. Midha

Department Chair

Dr. Sergio Felicelli 


\begin{abstract}
As the storage capacity of a given size of the hard disk drive continues to increase, the corresponding increase in the data storage density demands further reduction of the nano-scale gap (flying height) between the magnetic read/write head and the magnetic disk. In high-density magnetic recording, there can exist intermittent contact, and detection of contact also constitutes a necessary step in the process of thermal flying-height control. To further understand the head-disk contact phenomena, a computationally efficient fivedegree-of-freedom dynamic model was developed to simulate the motion of a magnetic head slider under the conditions of moving-bump collision and of contact due to an expanding protrusion on the slider for thermal flying-height control, with consideration of intermolecular (interatomic) forces. An integrated formulation for the Lennard-Jones potential including effects of the van der Waals forces was used to characterize the intermolecular forces between the magnetic disk and the magnetic head slider. This dynamic model was based on a stiffness matrix formulation involving the pitch angle, roll angle, height of the slider center of mass, the cross-track displacement of the slider and the cross-track displacement of the actuator arm, with a stiffness matrix obtained by combining those of the suspension and the air bearing. To improve the computational efficiency in calculating the resultant force and moments, the integration of the intermolecular forces was separated into a part with analytical and semi-analytical integration over the smooth
\end{abstract}


pad surface of the slider and another part of numerical integration in a small local region in the vicinity of a moving bump, which, in practice, could be a defect bump such as an adhered particle, or a laser bump usable in tests as a standard object with a known height. Compared to numerical results obtained without considering the intermolecular forces, these forces change the posture or attitude of the slider just before contact to create a slightly earlier contact when a moving bump on the disk tends to collide with the slider. This model was also used to simulate a stationary bump with an expanding bump height and with a zero lateral velocity so as to characterize an expanding protrusion on the slider surface in the active thermal actuation process for adjusting the flying height; the thermal expansion-related parameters used in this model were close to those extracted from a previous relatively complete fluid/thermo-mechanical analysis. The off-track motion of the slider was substantially increased by the intermolecular forces through a longer duration of contact, allowing prolonged application of the friction force under nonzero skew conditions. The numerical results obtained in this study shed light in the understandings of the precise behavior of the slider before, at, and after the contact with a bump or a thermal protrusion. 


\section{ACKNOWLEDGEMENTS}

The completion of this project could not have been possible without the guidance, advice, mentorship and cooperation of my advisor, Dr. Shao Wang. He has helped me solve problems in this thesis with his immense experience in the hard disk drive industry. I would like to thank Dr. Shing-Chung "Josh" Wong for co-advising me for my thesis.

I would like to thank Mr. Cliff Bailey for giving me an opportunity to tutor students on SOLIDWORKS and MATLAB, which helped me boost my technical and social skills.

I would like to thank the Department of Mechanical Engineering staff and faculty for their help during my study at The University of Akron, Ohio.

I would like to thank my parents Mr. Rajendra Pathak and Dr. Shobha Rajendra Pathak, and my sister for their support and advices. I would also like to thank my friends for their support during the Masters's Program 
TABLE OF CONTENTS

Page

LIST OF FIGURES.................................................. viii

\section{CHAPTER}

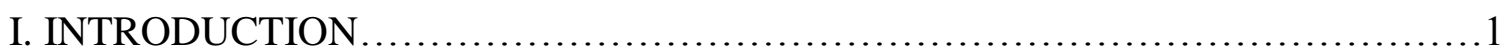

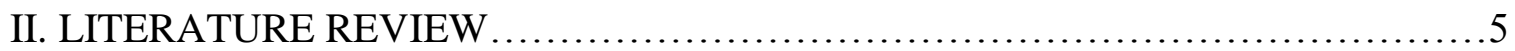

2.1 Five-Degree-of-Freedom (5DOF) dynamic system........................

2.2 Intermolecular forces.................................................

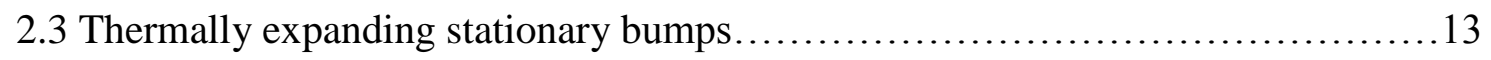

III. MODELLING OF THE DYNAMIC SYSTEM.............................17

3.1 Modelling the Five-Degree-of-Freedom (5DOF) system $\ldots \ldots \ldots \ldots \ldots \ldots \ldots \ldots \ldots \ldots$

3.2 Modelling the intermolecular forces....................................20

3.3 Theory on the thermally expanding stationary bump..........................35

3.4 Theory on the non-linear spring approach.............................. 38

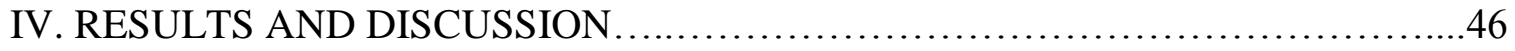

V. CONCLUSIONS .....................................................61

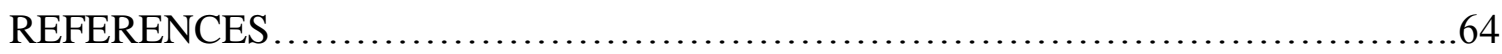


APPENDIX A: LIST OF SYMBOLS...................................... 70

APPENDIX B: PARABOLOIDAL BUMP FORMULATION .....................74

APPENDIX C: HAMAKER CONSTANT CALCULATION ........................78

APPENDIX D: RAW FIGURES FOR COMPARISON CASE ......................79

APPENDIX E: CALLING TREE'S FOR MATLAB PROGRAM.....................89 


\section{LIST OF FIGURES}

Figure $\quad$ Page

1.1 A typical HDD - Exploded view......................................

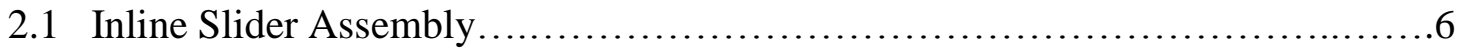

2.2 The working of AFM................................................. 12

2.3 Thermally Protruding Bump.......................................... 14

3.1 The Five-Degree-of-Freedom (5DOF) System........................... 18

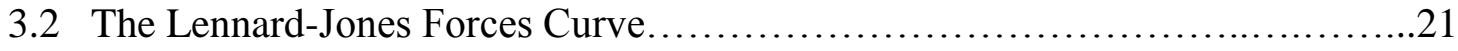

3.3 The division of the slider into many parts for simpler analysis................22

3.4 The division of the whole slider into smooth region and bump region..............23

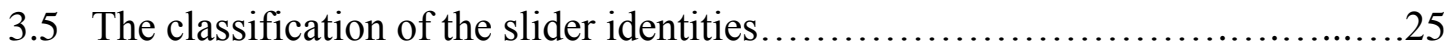

3.6 The theory behind the working of thermally expanding bump

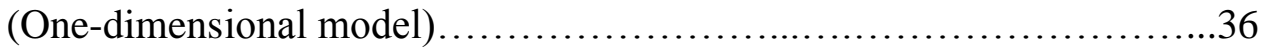

3.7 The heater function curve .............................................. 38

3.8 Different curves for contact theories...................................42

3.9 Validation of JKR nature of the research...............................45 
4.2 Response of the slider to single bump with a height of $20 \mathrm{~nm}$, a bump radius of $15 \mu \mathrm{m}$ and a skew, $\phi=10^{\circ}$

4.3 Maximum transverse displacement, $x_{\max }$,vs. the bump height $h_{b}$

4.4 Maximum normal contact force $F_{n, \max }$ vs. the bump height $h_{b} \ldots \ldots \ldots \ldots \ldots \ldots . .49$

4.5 Maximum transverse displacement $x_{\max }$ vs the radius of the bump $r_{b} \ldots \ldots \ldots \ldots 51$

4.6 The contact regime for a paraboloidal bump............................52

4.7 Maximum normal contact force $F_{n, \max }$ vs radius of the bump $r_{b} \ldots \ldots \ldots \ldots \ldots . \ldots 53$

4.8 Response for the slider for $h_{b}=12.5 \mathrm{~nm}, r_{b}=15 \mu \mathrm{m}$ and skew $\phi=10^{\circ} \ldots \ldots \ldots .54$

4.9 Maximum transverse displacement $x_{\max }$ vs. the skew angle $\phi$

4.10 Response Comparison for $20^{\circ}$ skew with and without Intermolecular forces

4.11 Maximum transverse displacement Xmax vs the coefficient of friction $\mu \ldots \ldots . .56$

4.12 Response of the slider with Intermolecular forces to multiple bumps

for bump height $h_{b}=12.5 \mathrm{~nm}$, bump radius $r_{b}=15 \mu \mathrm{m}$ and

skew angle $\phi=0$ under $\beta$-decoupled conditions

4.13 Response of the slider for a flat top bump with bump radius $r_{b}=5 \mu \mathrm{m}$, bump height $h_{b}=18 \mathrm{~nm}$ and skew angle $\phi=10^{\circ}$ under the influence of intermolecular forces.... .58 
4.14 Comparison of cases for a flat top bump with and

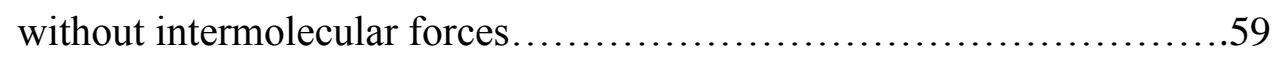

4.15 Response of the slider to the expansion and contraction

of a thermally actuated protrusion for $h_{b s}=22 \mathrm{~nm}$ and

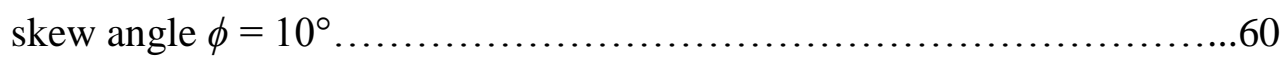




\section{CHAPTER I \\ INTRODUCTION}

Since the invention of the computer, storing memory has been a very important task in this industry. The first hard disk drive was made by IBM in 1956. It was named as 350 RAMAC. It was as big as two refrigerators kept side by side. The maximum size it could store was $3.75 \mathrm{MB}$ over a stack of 50 disks or platters. Since then, the technology has advanced at a very fast pace. As of today, we have hard disk drives which can store up to $10 \mathrm{~TB}$ with a form factor of 3.5-inch. This rapid development in technology needed a lot of work in the improvement of reading mechanics of the HDD. In order to appreciate the work, we need to know the working of the HDD.

Another alternative way for storage today is cloud storage. It is also fast emerging technology which enables us to store data in a virtual hard disk drive via the internet. The way it works is, service provider companies maintain physical servers which are responsible for storing the data and make it available anywhere and anytime. The data is stored in many HDDs places on racks in buildings similar to the warehouse, sometimes called the storage banks. These servers work 24/7 which require very sophisticated facilities. There are many advantages like convenience in accessing the data from any place and any device, it is maintenance free, it speeds up backup and restore process etc. But there are many dis-advantages like the security of the data stored is vulnerable to hacks, it needs high speed internet to upload larger data etc. The cloud storage demands high 
capacity and fast access HDDs. Another application of fast access and high capacity HDD is Automatic Teller Machines (ATMs). ATMs need to access the data of the user in fraction of time and then respond back to the user with their corresponding data. For this purpose, they require very fast access HDDs and large capacity HDD to store the data of millions of users. Hence in the present age, we require these type of hard disk drives to meet the demands of the market.

The typical hard disk drive today has the following parts. It has a magnetic disk mounted on a spindle. The spindle gets its motion by a step motor present in the assembly. The data reading is done by the slider and pad assembly which is mounted onto the actuator arm. The actuator arm is connected to a second stepped motor which controls the movement of the arm. The whole electronics involved here is managed by a disk controller chip which has printed circuit cables connecting all the electronics. The amount of data stored in given area on the disk is known as "Areal Density". For achieving the high storage capacity in HDDs with the existing form factor, we need to achieve higher areal density. This is being done by lowering the flying height of the slider. The flying height of the slider is being lowered to height below $10 \mathrm{~nm}$. Hence the concept of nano-spacing is very important and is the emphasis of the current research. The figure 1.1 shows a hard disk drive and its parts.

The actuator of the HDD is a permanent magnet, controlled by a voice coil motor, which helps in data reading and writing. The HDD works on the principle of magnetic data storage. The platter is a non-magnetic material, usually made of aluminum alloys, glass or ceramics, which is coated with a thin layer (10-20 nm thickness) of a magnetic material by a sputtering process. In magnetic sputtering, the thin platters are passed through a chamber 
in which the magnetic material is a sprayed onto the platter. It is a controlled process as the precision is very important. After the magnetic material is sprayed, layer of diamond like carbon (DLC) is coated onto which a polymer lubricant layer is sprayed for reducing the air bearing friction.

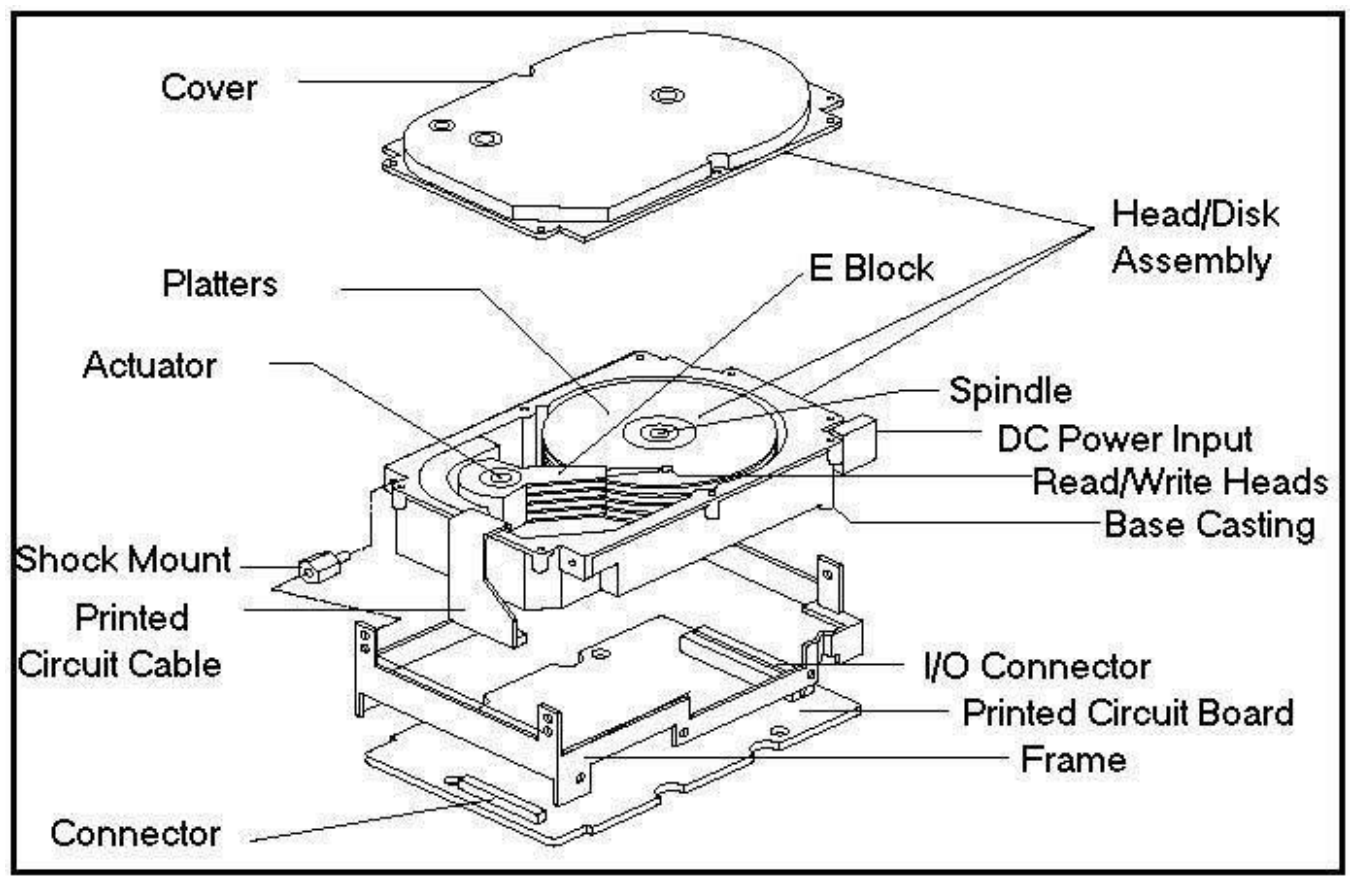

Figure 1.1 A typical HDD - Exploded view

Usually the manufacturing of the platters are very precise but there are some abnormalities some times. There are small asperities on the bump surface which affect the reading/writing ability of the slider. To study the effect of such asperities, lots of studies have been implied.

The purpose of this research is to study the contact mechanics associated with the collision model of the slider when a paraboloidal bump comes in contact with the slider. Because, we are dealing with the flying heights in the range of $10 \mathrm{~nm}$, we can also see the effects of intermolecular forces. We integrate the intermolecular forces model with the 
collision model. In recent studies, the flying height is tested using a thermally expanding bump. We simulate the same, and integrate this with the previous models.

This research is an analytical and numerical study of the behavioral mechanics of the slider arm assembly when a paraboloidal (see APPENDIX B for formulation) bump on the disk, comes in contact with the slider head, under the effects of intermolecular forces. It also studies the effect of intermolecular forces when a thermally expanding bump is developed on the slider. This is achieved using the multi-paradigm numerical computing software, MATLAB. 


\section{CHAPTER II}

\section{LITERATURE REVIEW}

The slider and actuator arm assembly is modelled numerically in order to achieve the goals of the research. Researchers have taken many different approaches to study and simulate the behavior of the slider arm assembly.

\section{$\underline{\text { 2.1 Five-Degree-of-Freedom (5DOF) dynamic system }}$}

The study started with a simple single degree of freedom by Ono et al., analyzing the bouncing vibrations of a contact recording head slider. The disk surface was modelled as harmonic and wavy. They have discussed the relationship between the bouncing height and the frequency of the waviness. They emphasized on the importance of the complete tracking without separation. They calculated the complete tracking conditions of the disk waviness under disturbances for various design parameters values like waviness phase under the disturbances of ten nanometers bounce height [1].

In other studies, Yoneoka et al. experimentally analyzed the dynamics of the inline

flying-head assembly using a laser Doppler anemometer and laser Doppler vibrometer (LDV) and deduced that lateral and torsional modes of the suspension vibration were caused by access-direction acceleration of the rectangular wave and noticed spacing fluctuations, and pivot slip due to the lateral vibrations. They performed experiments 
studying the flying stability, modal analysis and time response to access direction acceleration [2].

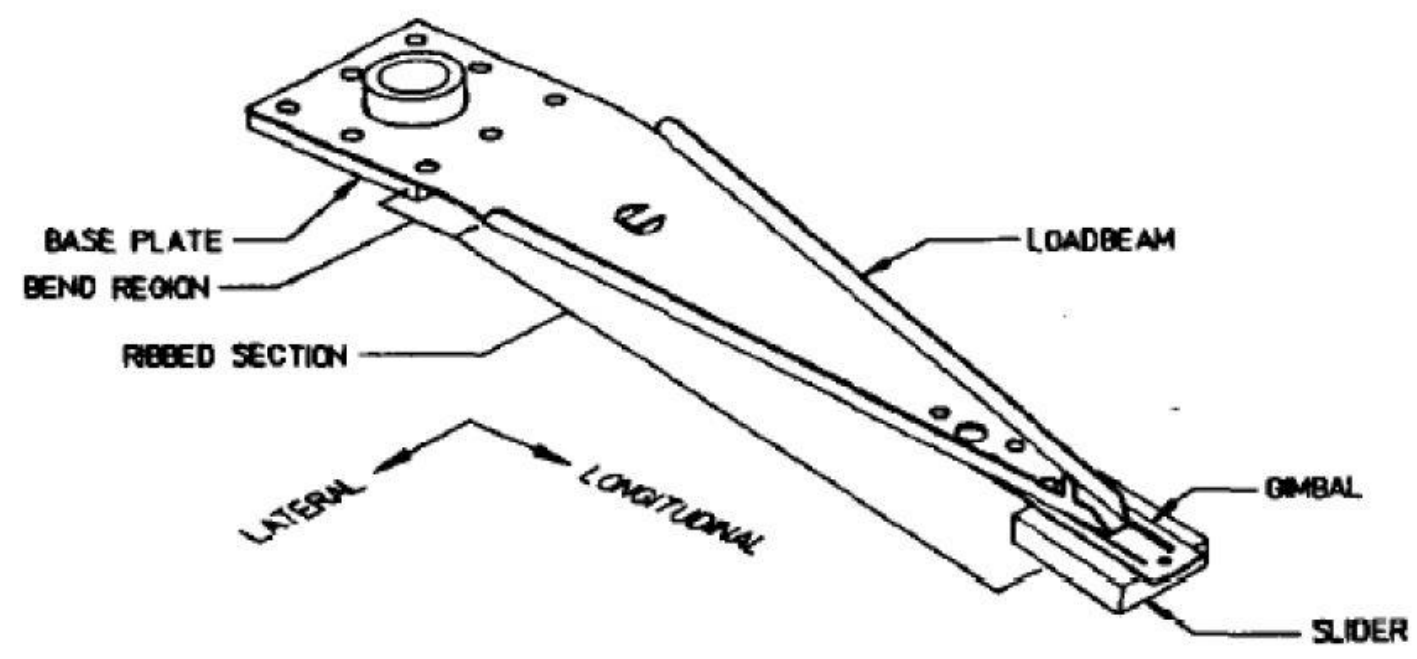

Figure 2.1 Inline Slider Assembly [34]

Ono et al. used a 2-DOF model with linear air bearing springs to conclude that the bouncing vibration of a slider in a near contact regime is a self-exited vibration due to the combination of adhesion and friction force. They showed that, by reducing the ambient pressure the slider began to touch down and exhibited bouncing vibrations and continued till it reached an ambient pressure higher than touchdown pressure. They experimentally concluded that the bouncing vibration is a self-excited vibration of the lower natural frequency of the slider and the air-bearing system. Destabilizing effects were generated from the interfacial force at contact air-bearing pad and the disk surface. They observed a hysteresis phenomenon of different touch-down and take-off points when decreasing and increasing the nominal flying height due to nonlinear nature of the contact force. They 
concluded that the bouncing vibration of a slider is caused by the combination of an adhesion force and contact force [3].

A 2-DOF tripad approach was implemented by Iida and Ono et al. who numerically investigated the tracking ability, dynamic contact and frictional forces, studying the effects of front and rear air bearing stiffness, nominal flying height, and coefficient of friction on tracking ability and contact force. They concluded that the spacing variations were caused by slider dynamics, micro-waviness of the disk surface and the distance of the contact pad position from the rear air bearing center [4].

In a similar study Ono and Takahashi used a two-degree-of-freedom tripad contact slider to analyze the bouncing vibrations over a harmonic wavy platter surface. They found that the bouncing vibrations could be classified as contact resonance and impact vibration and they studied their occurrences. They noticed that contact damping significantly decreases the bouncing height [5].

Track misregistration occurs when there are off-track jitters on the head-gimbal assembly. This phenomenon was studied by Wang et al. They concluded this phenomenon was caused by airflow inside the hard disk drive (HDD) and disk velocity and suspension type of the assembly were important factors affecting the reading/writing ability of the head gimbal assembly (HGA). They deduced that the contact between HGA and disk surface accelerated the off-track jitter and vertical spacing modulation. They used LDV to find that the first torsional mode (T1) and the first bending mode (B1) of the suspension were the primary contributors to the off track jitter [6]. 
Studying the contact mechanics of a nano-slider, Cha and Body numerically simulated the interactions of the slider using a Hertzian contact model. They modelled multiple asperities on the platter numerically using a combination of sinusoidal waviness and a Gaussian roughness. They calculated the contact force using radius of the asperities, and they showed that their results were correlating with the impulse momentum based contact model [7].

In a similar study, Ono and Nakagawa experimentally studied the dynamic contact forces and meniscus forces originated from bouncing motion of the hemispherical sliders on the magnetic disks. It was found that the maximum contact force per penetration depth can be estimated by the Hertz contact theory. They concluded that the maximum dynamic adhesion force is almost equal to the static adhesion force of the slider [8].

As the technology has advanced, the size of the slider has gotten smaller. The sizes of sliders have gone from micro to femto (current advancement). Of these, pico sliders are the most used sliders. Many tests were performed on these sliders and the performance was improved. One of such investigations was done by Kohira et al. observing the dynamic behavior during the slider disk contact. Their study was an emphasis on the optimum lubricant thickness for proximity recording. They used LDV to deduce that the roll and pitch frequencies were a function of the frequency of contact and disk velocity, and these frequencies of the air bearing increased with decreasing disk velocity [9].

Attaining higher areal recording density has always been the primary focus of the researchers in this field. In order to obtain 1TBit/in. ${ }^{2}$ active research is going on. One of them was done by Chen and Bogy, who numerically simulated the bouncing vibrations of an air bearing slider in near of partial contact. They used three different dynamics models 
for the analysis. They deduced that the contact, adhesion and frictional forces were not the direct causes for the bouncing vibrations of the slider. The simulation results showed that the slider's bouncing vibration was a forced vibration caused by the moving disk microwaviness and roughness, which excited the bouncing vibrations of the partial contact slider [10].

Yuan and Wang developed a five degree of freedom model to simulate a pico slider system. They numerically simulated a paraboloidal bump and studied the contact mechanics of the system. As their model also included the transverse displacement, they were able to predict the off-track motion. They showed various case studies comparing the slider behavior for different conditions and validated it with the real time scenario. They concluded that the maximum transverse displacement of the slider increases with the coefficient of friction and the skew angle. They noticed that increasing the rotational speed of the disk decreases the transverse displacement due to shorter contact time. The contact time ratio (ratio of contact time and period of contact force) for multiple bumps was big when there were multiple bumps in a sequence which lead to increased average normal contact force, and hence, the greater transverse displacement. They observed that the roll angle contributed to the transverse displacement and that the off-track motion of the slider was dominated by the rotational mode of the actuator arm and the suspension sway mode [11].

\subsection{Intermolecular forces.}

The major objective of the present research is numerically modelling of the dynamic behavior of the head-disk interface including stability. Usually not all the parameters are taken into consideration while modeling the system and often the 
intermolecular forces are neglected to be a part of a study. Due to the very constricted space, there might be intermolecular forces between the slider and the magnetic disk. Researchers have started to consider such conditions in order to get the real life simulations.

Wu and Bogy numerically studied the effect of intermolecular forces on the air bearing sliders at a flying height of $5 \mathrm{~nm}$. They discretized the slider surface into unstructured triangles and found the intermolecular forces between each triangular cell of the slider and disk surface. They studied the contributions of the intermolecular forces to the contact force, pitch and roll moments. They found that the van der Waals forces affected the flying height and the pitch angles for both positive pressure sliders and negative pressure sliders [12].

Li et al. have done similar work in emphasizing the effects of intermolecular forces on deep sub-10 nm spaced sliders. After their simulations, they have concluded that the intermolecular forces affect the flying height of the slider when the flying height goes lower than the $10 \mathrm{~nm}$ mark. They proposed a new femto slider design which could help in reducing the effects from the intermolecular forces [13].

Studying the nonlinear dynamic analysis of the head disk interface, Thornton and Bogy worked on the instability of head disk interfaces due to intermolecular forces. In their study, they have shown that the slider can be forced into unstable motions, and the intermolecular forces can explain the phenomenon like flying-height hysteresis, intermittent instability and snapping from stable to unstable proximity. They concluded that multiple equilibria exist at sub-7 $\mathrm{nm}$ flying height regime [14]. 
In a further study, Thornton and Bogy worked on the nonlinear dynamics analysis of the head-disk interface by including the intermolecular adhesion forces on a sub-5 nm flying air bearing sliders. Their parametric study showed the dependence of the stability of the slider on the variables, as they found way to achieve guidelines for the air-bearing surface and disk morphology system design. It was found that the head-disk interface could become unstable due intermolecular forces below the flying height of $6 \mathrm{~nm}$. They inferred from their studies that, by minimizing the intermolecular adhesion forces and the flyingheight modulation and maximizing the air-bearing stiffness and damping, maximum stability could be achieved [15].

Ono et al. experimentally concluded that the adhesion force between the slider and magnetic disk was caused by the formation of a meniscus although the slider was vibrating and the disk was stationary. Any attractive force enough to generate a clear velocity drop could not be detected unless the meniscus was formed [3].

Deoras and Talke used a single degree-of-freedom spring mass damper model to simulate the dynamic response of a typical magnetic recording slider under the effect of intermolecular forces. They studied the impulse response of the model to show that the slider could snap even at flying heights greater than the critical height. They concluded that the intermolecular forces caused the reduction of the flying height due to the additional attractive forces on the slider [16].

Despite the weak nature of the intermolecular forces, it has become a key component for the working of the Atomic Force Microscope (AFM). AFM's are a variant of scanning probe microscopy which are used to magnify objects in the orders of fractions of nanometers in magnitude. It was invented by C.F Quate, Ch. Gerber, and G. Binnig in 
1995, as an improvement of the scanning-tunneling microscope (STM) [40,41]. AFM has five important components, a sharp tip which is mounted onto a cantilever spring, a deflection detecting mechanism, feedback mechanism which controls the deflection, a piezoelectric mechanical system which moves the sample with respect to the tip, and a display system which converts the raw data into an image. Figure 2.2 shows the working of the AFM [42].

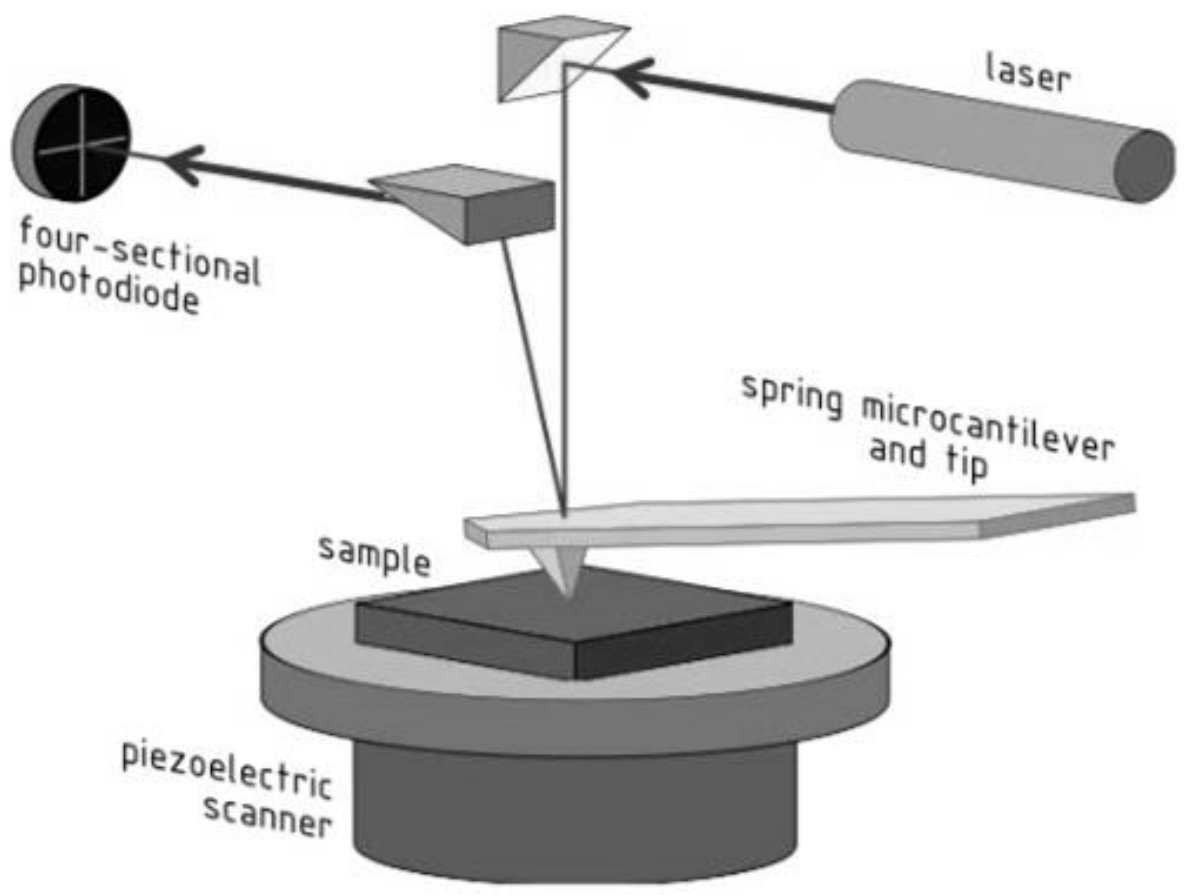

Figure 2.2 The working of AFM

The AFM provides a 3D profile of the surface on a nanoscale by measuring the forces between the probe and the sample surface. The tip touches the surface gently and records the intermolecular forces between the surface and tip [42]. Applications AFM include biochemistry applications (imaging the structure of biological molecules, cellular components, cells or tissues), chemistry, materials science and nanotechnology 
applications (imaging of polymers, nanostructures or other materials) and physics and biophysics applications (measuring forces between the AFM tip and the sample surface).

\subsection{Thermally expanding stationary bumps:}

As the areal density of the hard disk drives have been increasing competitively, the flying height (gap between the head and disk) decreased continuously. To reach an areal density of $1 \mathrm{Tbit} / \mathrm{in}^{2}$, it was proposed that the flying height must be in the order of $2.5 \mathrm{~nm}$. To reach this low spacing, we would require a valid way to measure the flying height and control it. One of the methods being implemented is using a thermally expanding bumps to detect and control the flying height. The work done in this regards is discussed in this section.

Kurita et al. simulated the heat transfer in the slider using a finite element model and obtained the thermal deformation of the air bearing surface. It was observed that the protrusion magnitude was large enough to control the flying height and was proportional to the heater power. They fabricated a thermal nanoscale actuator using thin film processing and showed a linear reduction in the magnetic height as electric power was applied to this actuator. They concluded that there was no significant impact on the reliability of the read element [17].

Wang et al. measured the head temperature as a function of the write current, frequency and flying conditions. They observed significant cooling effects when the slider was flying on the disk. Their experimental data of temperature coefficients validated the simulation results. The amount of head protrusion due to the write current as determined 


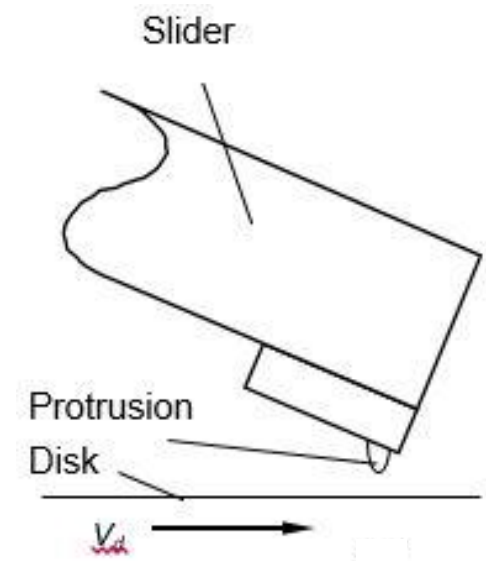

Figure 2.3 Thermally Protruding Bump

from the touchdown height matched the atomic force microscope data. They deduced that as long as the protrusion did not create interference with the disk during flying, the flyability was not affected, excessive contact during the recording process could cause offtrack jitter and tracking errors [18].

Ju reported a combined experimental and numerical simulation study of selfheating in the thin-film magnetic recording head due to write currents. An important factor affecting the magnitude and spatial distribution of the temperature rise in the magnetic head was found to be the magnetic saturation of the yoke structure of the writing current. The results showed that the heat transfer across the air bearing had a strong influence on the temperature rise in the write heads. The time-averaged heat generation rate due to write current pulses was estimated and the spatial heat generation profiles were modelled by analyzing the small-signal electric impedance spectra [19].

Nikitin et al. studied the spatial and temporal profiling of the protrusion in magnetic recording heads. They observed that the thermomechanical properties of the head materials 
affect the magnetic performance and that the heat produced by the write head affects mechanical performance. They experimentally concluded that more effort were needed to reduce the Joule heating rather than reducing the eddy currents or magnetic hysteresis loss [20].

$\mathrm{Xu}$ et al. performed a thermal analysis of a magnetic head. The temperature distribution on the air bearing surface was mapped to display a butterfly shaped pattern around the head elements. The distribution was useful to understand the head thermal protrusion and to design the head structures. They concluded that the temperature distribution across the head was very narrow when the slider was flying, in comparison to the case when the slider was not flying. The temperature increase was uniform in every study condition. The increase rate was found to be $1.46^{\circ} \mathrm{C}[21]$.

Aoki et al. numerically and experimentally described the differences between the two boundary conditions, namely, natural convection off the disk and flying on the disk. They used finite element models and quantitatively identified the differences in the heat path for the two boundary conditions. They verified the numerical predictions with the experimental measured results [22].

Pust et al. developed a three dimensional finite element thermomechanical model of the head surface which showed that the distribution of the thermal stresses, caused by the mismatch of coefficient of thermal expansion, changed completely close to the air bearing surface due to the protruded head surface. For a photoresist coil insulator, it was found that the Poisson's ratio also had an impact on the deformation of the surrounding transducer apart from the coefficient of thermal expansion (CTE) and Young's Modulus. They noticed that the mismatch of CTE between the constituent materials in the head 
caused large relative local displacement and stress distributed throughout the volume. These stresses became compressive with depth onto the transducer from the air bearing system due to larger CTE of metallic shields. They concluded that the ambient temperature of the head in the operation was largely dependent on the power dissipated within the writer circuit [23].

Investigating the effects of the air bearing surface design on the thermal actuation, Juang and Bogy created a three dimensional finite element model of an entire slider with a detailed read/write transducer structure and conducted thermal-structural coupled-field analysis of velocity slip and temperature jump boundary conditions to formulate the heat transfer across the head-disk interface when a slider flies over a spinning disk. It was found that the effect of the pressure was more significant than the flying height on the heat conduction from the slider to the disk. They proposed a new design of a slider, which had $100 \%$ virtual efficiency with less power consumption [24].

As the technology advances, we need larger capacity hard disk drives to store data in the present form factor. In order to do that, it is necessary to decrease the flying height to increase the areal density. For a nanoscale spacing, it is important to consider the effects of intermolecular forces as these play an important role in the dynamics of the slider. This is done by combining the effects of intermolecular forces with the simplified five-degreeof-freedom model of the slider arm assembly. Similarly, for controlling the flying height of the slider, we study the effects of a thermally expanding bump on the reading element of the slider and quantify its behavior. Finally, for recreating a real environment, we combine the intermolecular model with the thermally expanding bump model and perform case studies on these. 


\section{CHAPTER III MODELLING OF THE DYNAMIC SYSTEM}

The study in this research was accomplished by modelling the system in three steps. The first step was to model the five-degree-of-freedom (5DOF) system which would simulate the collision of the slider with a bump. Then we modeled a system which could simulate the intermolecular forces due to the tight spacing of the system, and then integrated it with the 5DOF system. Finally, we modeled a thermally protruding bump, controlled by a virtual heater current simulating an on/off switch. We integrated this with the previously integrated model in order to achieve the goals of the research.

\section{$\underline{3.1 \text { Modelling the Five-Degree-of-Freedom (5DOF) system }}$}

We modeled a 5DOF system as shown in the figure 3.1. It was modelled in a way such that the actuator arm could have transverse displacement, the slider could have transverse displacement, and the actuator arm could be displaced vertically through its center of mass, while the arm could undergo pitch and roll. When there is no contact in the model, it has the following equation of free vibration:

$$
M \ddot{X}+C \dot{X}+K_{C} X=F
$$

where 


$$
X=\left[x_{\text {arm }}, x, z, \theta, \beta\right]^{T},
$$

here $x_{\text {arm }}$ is the transverse displacement of the actuator arm, $x$ the transverse (off-track) displacement of the slider, $z$ the vertical displacement of the center of mass of the slider, $\theta$ is pitch angle, $\beta$ is roll angle, $M$ is the mass matrix, $C$ is the damping matrix, $K_{c}$ is the combined stiffness matrix for the suspension and air bearing [11] defined as follows,

$$
\left[K_{c}\right]=\left[\begin{array}{ccccc}
k_{\text {arm }}+K_{11} & -K_{11} & 0 & 0 & 0 \\
-K_{11} & K_{11} & 0 & 0 & K_{14} \\
0 & 0 & K_{22}+k_{11} & K_{23}+k_{12} & k_{13} \\
0 & 0 & K_{32}+k_{21} & K_{33}+k_{22} & k_{23} \\
0 & K_{41} & k_{31} & k_{32} & K_{44}+k_{33}
\end{array}\right] \text { Eqn 3-3 }
$$

where, $K_{c}$ is formed by the combination of equivalent stiffness matrix of the actuator arm in the transverse direction, $k_{\text {arm, }}$, the air bearing stiffness matrix $[k]$, and the suspension stiffness matrix $[K]$.

$$
[M]=\left[\begin{array}{ccccc}
m_{11} & 0 & 0 & 0 & 0 \\
0 & m_{22} & 0 & 0 & 0 \\
0 & 0 & m_{33} & 0 & 0 \\
0 & 0 & 0 & m_{44} & 0 \\
0 & 0 & 0 & 0 & m_{55}
\end{array}\right]
$$

here $M$ is the mass matrix, and $[C]$ can be found in the same way as $\left[K_{c}\right]$.

$$
F=\left[0, F_{t}, F_{n}+F_{i}, M_{\theta}+M_{i x}, M_{\beta}+M_{i y}\right]^{T}
$$


where $F_{t}$ is the friction force in the transverse direction, $F_{n}+F_{i}$ is the total normal force acting on the slider due to the contact force and the intermolecular forces, $M_{\theta}+M_{i x}$ is the total pitch moment due to the contact and intermolecular forces, and $M_{\beta}+M_{i y}$ is the total roll moment due to the contact and intermolecular forces [11,34].

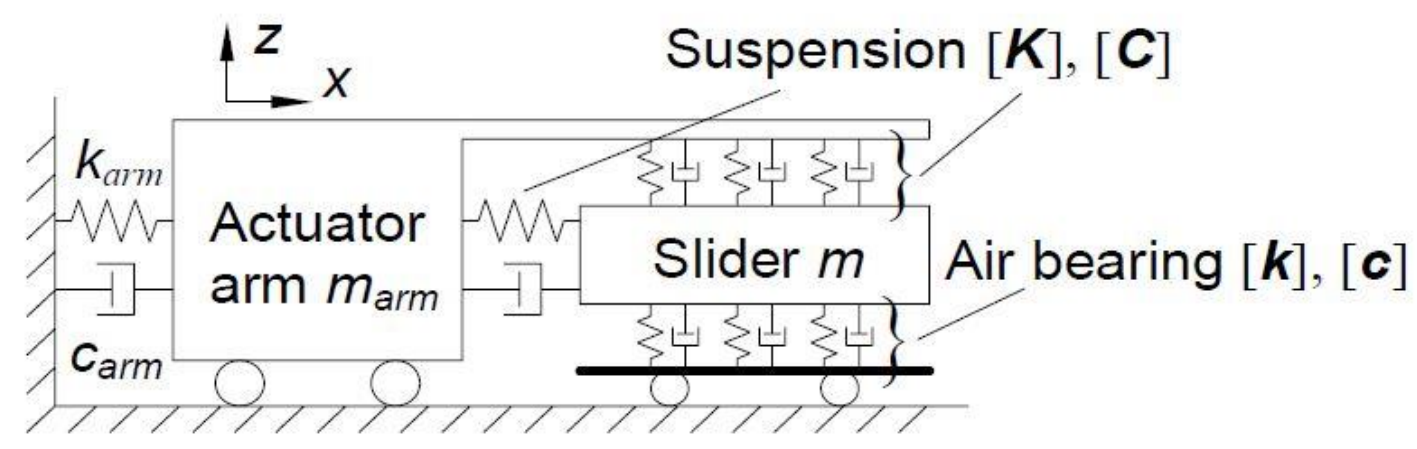

Figure 3.1 The Five-Degree-of-Freedom (5DOF) System [11,34]

The model was achieved using MATLAB differential equation solver, ode45, which uses a variable step Runge-Kutta Method to solve differential equations numerically [25]. To solve the above equation, we formulated ten different equations which calculated the velocity and acceleration from the given displacements. The first two set of equations represented the actuator arm, $x_{\text {arm }}$, the third and fourth represented the transverse displacements of the slider, $x$, the fifth and sixth represented the vertical displacement of the slider, the seventh and eighth equations represented the pitch angle and the ninth and tenth represent the roll angle.

This research was an extension to the work done by Yuan and Wang [11,34], who used this 5DOF system to predict and measure off-track motion of the slider. Their model was modified to obtain our research goals. 


\subsection{Modelling the Intermolecular Forces}

The main objective of this research was to predict the behavior of the slider under the influence of intermolecular forces. The significance of the intermolecular force was essential because the form factor of the problem was so small that they had an impact on the study. The van der Waals forces between the disk and slider tend to pull the slider towards the disk. This increased the normal contact force and the transverse displacement of the slider.

There are four basic types of forces in nature. They are strong and weak interactions between protons, neutrons and elementary particles, electromagnetic forces and gravitational forces. Interatomic forces is one variant of the electromagnetic forces. These forces are the basis for the phenomenon such as adhesion, surface tension, physical adsorption, wetting etc. The general properties of these forces are

- $\quad$ These are forces acting at a very small distances (in the order of $10 \mathrm{~nm}$ ).

- These can be either attractive, repulsive or both depending upon the application.

There are different types of intermolecular forces. They are dipole forces, dipoledipole interactions, London dispersion forces [27,37].

In this current research, to explain the effect of van der Walls forces, we used the Lennard-Jones Potential equations. It is given as follows:

$$
\phi_{L J}=-\frac{C}{r^{6}}+\frac{D}{r^{12}}
$$


where $C=10^{-17} \mathrm{Jm}^{6}$ and $D=10^{-134} \mathrm{Jm}^{12}$. Here $C$ and $D$ proportionality constants in vacuum $[16,26,37]$. The negative regime is for the attractive forces and the positive regime is for repulsive forces. In order to get the intermolecular forces, the above equation is integrated over the slider volume, which are discretized into small elements.

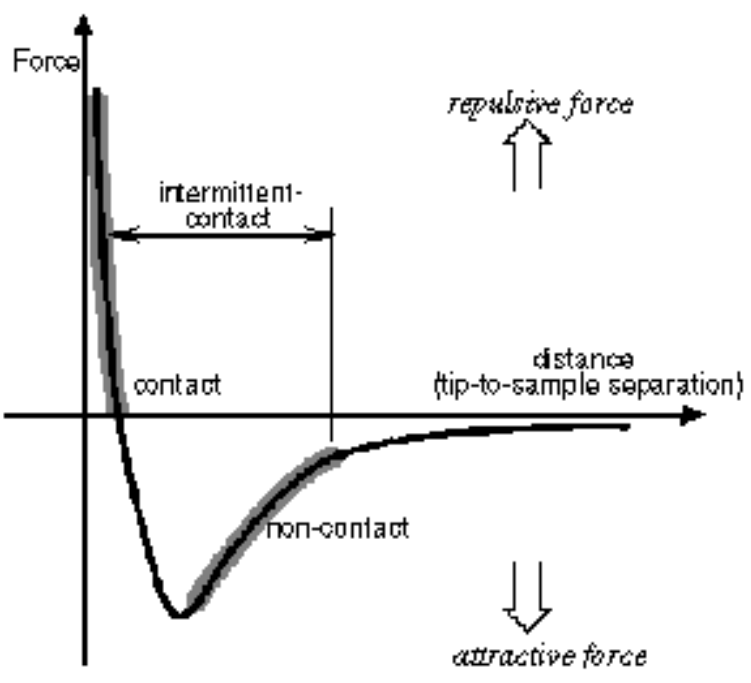

Figure 3.2 The Lennard-Jones Forces Curve

The equation for a single element over the slider volume is given by

$$
F_{i}=\frac{A}{6 \pi} \iint \frac{d x d y}{h^{3}}-\frac{B}{45 \pi} \iint \frac{d x d y}{h^{9}}
$$

where A is the Hamaker's Constant, B is a constant and $\mathrm{h}$ is the separation between the slider and the disk. The above equation was tested to check whether the obtained results were in correlation with the standard lennard jones force curve (see figure 3.2). After tests, we obtained the following figure which supports the lennard jones force curve. 
For modelling the intermolecular forces, initially the quad2D module of the MATLAB software was used, which numerically evaluates double integrals using a tiled method [25]. It could numerically evaluate a function in 30,000 iterations max. This limited our study at certain locations of the slider, particularly in the area of contact of the bump with the slider. So solve this problem, the slider was later divided into many smaller parts manually as shown in the figure 3.3 , where regions 1,2 , 4 , and 5 represented the noncontact regions and region 3 represented the contact region, and then integrated using the quad2D module. This approach narrowed down the area of contact to a restricted boundaries. Same problems were encountered which then could have been solved by loosening the tolerances. But for the accuracy purposes, a new approach was used.

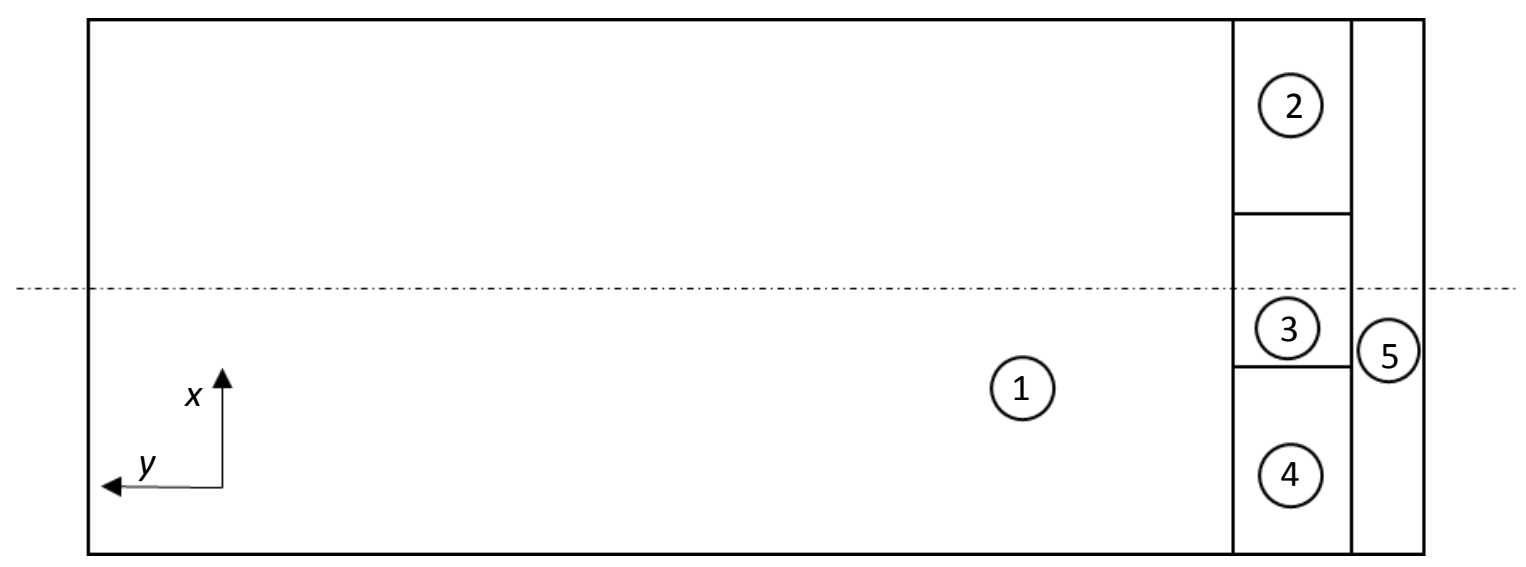

Figure 3.3 The division of the slider into many parts for simpler analysis

From the above numerical results, it was concluded that the code failed to compute the intermolecular forces only in the area where there is a contact of the slider with the bump. The time for computation was long as we had numerous functions to solve in a single iteration. To overcome these difficulties, the slider was divided into two different regions, namely, smooth region - the region including the whole slider excluding the area of bump 
contact and the bump region - the region of bump contact. Overall, three methods of computations were used. They were:

1. Analytically solving the intermolecular forces equation which could be used directly for computation.

2. Semi-analytically solving the moment about $x$ axis

3. Semi-analytically solving the moment about $y$ axis.

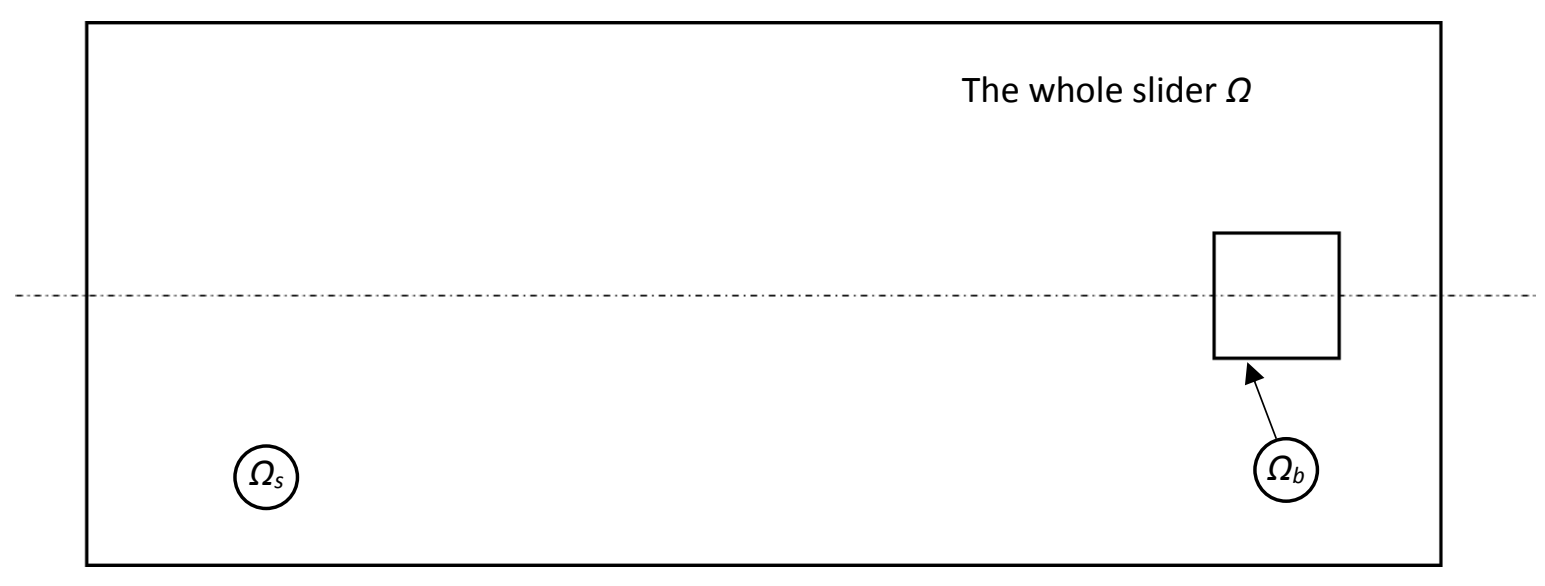

Figure 3.4 The division of the whole slider into smooth region and bump region

$$
\begin{array}{ll}
\iint_{\Omega} q d x d y=\iint_{\Omega_{1}} q d x d y+\iint_{\Omega_{b}} q d x d y & \text { Eqn 3-8 } \\
=\iint_{\Omega_{1}} q_{s} d x d y+\iint_{\Omega_{b}} q_{s} d x d y+\iint_{\Omega_{b}}\left(q-q_{s}\right) d x d y & \text { Eqn 3-9 }
\end{array}
$$




$$
=\iint_{\Omega_{1}} q_{s} d x d y+\iint_{\Omega_{b}}\left(q-q_{s}\right) d x d y
$$

where, $q$ is the function of for finding the intermolecular forces $F_{\mathrm{i}}, \Omega$ is the total area of the slider, $\Omega_{1}$ is the area of the smooth slider and $\Omega_{\mathrm{b}}$ is the area of contact of the bump with the slider. To minimalize the computation time, the intermolecular forces computed for the smooth slider was analyzed using analytical and semi-analytical integrated function over the smooth surface. We obtained the analyzed smooth slider equation in the given below fashion.

In the equation $3-7$, the negative term is to the order of negative nine (-9), and hence when we integrate that term we get a very small term which is very negligible. Hence, this term was neglected. So we were left with the following equation.

$$
F_{i}=\frac{A}{6 \pi} \int_{\frac{-w}{2}}^{\frac{w}{2}} \int_{\frac{-L}{2}}^{\frac{L}{2}} \frac{d x d y}{h^{3}}
$$

where,

$$
h=z+\theta y-\beta x-\frac{H}{2}
$$

here $w$ is the width of the slider, $L$ is the length of the slider, $z$ is the vertical displacement of the center of mass of the slider in meters, $\theta$ is the pitch angle in radians, $\beta$ is the roll angle in radians and $H$ is the slider thickness in meters as shown in the figure 3.5. 


$$
F_{i}=\frac{A}{6 \pi} \int_{\frac{-w}{2}}^{\frac{w}{2}} \int_{\frac{-L}{2}}^{\frac{L}{2}} \frac{d x d y}{\left(z+\theta y-\beta x-\frac{H}{2}\right)^{3}}
$$
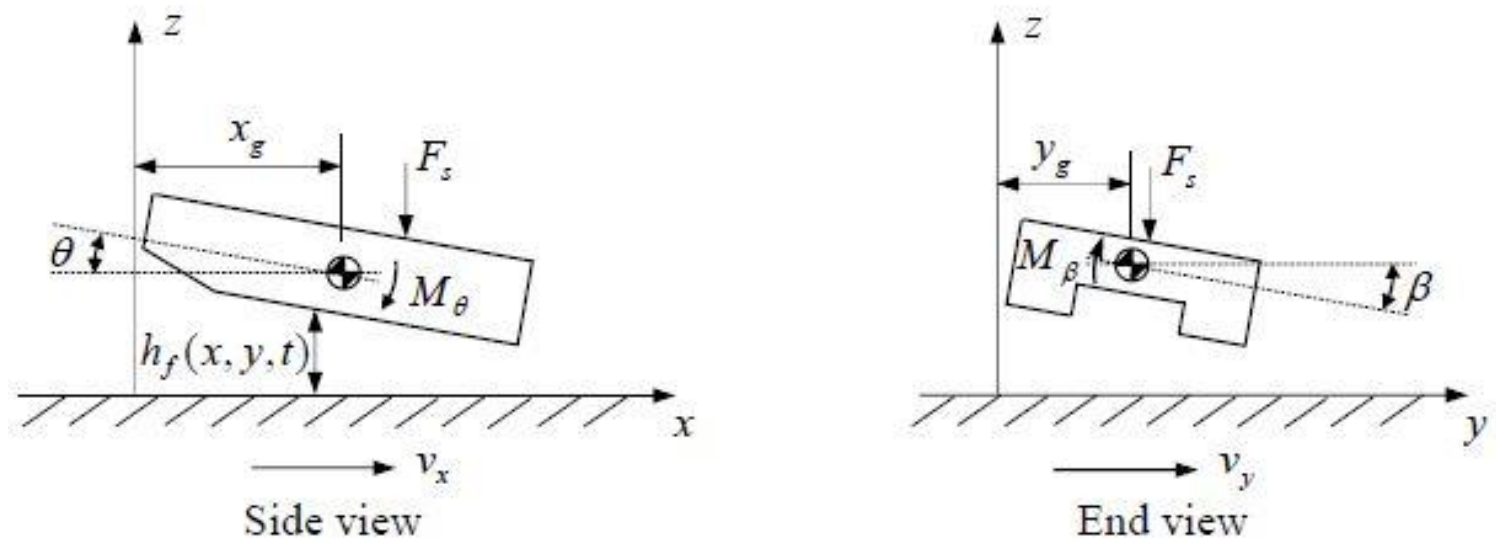

Figure 3.5 The classification of the slider identities [34].

First integrating with respect to $\mathrm{x}$

$$
\begin{gathered}
I_{1}=\int_{\frac{-w}{2}}^{\frac{w}{2}} \frac{d x}{\left(z+\theta y-\beta x-\frac{H}{2}\right)^{3}} \\
I_{1}=\int_{\frac{-w}{2}}^{\frac{w}{2}} \frac{d x}{\left(-\beta x+\left(z+\theta y-\frac{H}{2}\right)\right)^{3}}
\end{gathered}
$$

If $\beta=0$, 


$$
\begin{gathered}
I_{1}=\int_{\frac{-w}{2}}^{\frac{w}{2}} \frac{d x}{\left(z+\theta y-\frac{H}{2}\right)^{3}} \\
I_{1}=\frac{1}{\left(z+\theta y-\frac{H}{2}\right)^{3}} \int_{\frac{-w}{2}}^{\frac{w}{2}} d x \\
I_{1}=\left[\frac{x}{\left(z+\theta y-\frac{H}{2}\right)^{3}}\right]_{w / 2}^{-w / 2}=\frac{w}{\left(z+\theta y-\frac{H}{2}\right)^{3}}
\end{gathered}
$$

If $\beta \neq 0$

$$
\begin{gathered}
I_{1}=\left[\frac{1}{\left(2 \beta\left(z-\beta x+\theta y-\frac{H}{2}\right)\right)^{2}}\right]_{-\frac{w}{2}}^{\frac{w}{2}} \\
I_{1}=\frac{1}{2 \beta\left(z-\beta \frac{w}{2}+\theta y-\frac{H}{2}\right)^{2}}-\frac{1}{2 \beta\left(z-\beta\left(\frac{-w}{2}\right)+\theta y-\frac{H}{2}\right)^{2}}
\end{gathered}
$$

Let $a=z+\theta y+H / 2$ 


$$
\begin{gathered}
I_{1}=\frac{1}{2 \beta\left(a-\beta \frac{w}{2}\right)^{2}}-\frac{1}{2 \beta\left(a+\beta \frac{w}{2}\right)^{2}} \\
I_{1}=\frac{\left(a+\beta \frac{w}{2}\right)^{2}}{2 \beta\left(a-\beta \frac{w}{2}\right)^{2}\left(a+\beta \frac{w}{2}\right)^{2}}-\frac{\left(a-\beta \frac{w}{2}\right)^{2}}{2 \beta\left(a+\beta \frac{w}{2}\right)^{2}\left(a-\beta \frac{w}{2}\right)^{2}}
\end{gathered}
$$

From mathematical properties

$$
\begin{gathered}
(A+B)(A-B)=A^{2}-B^{2} \\
I_{1}=\frac{\left(a+\beta \frac{w}{2}\right)^{2}-\left(a-\beta \frac{w}{2}\right)^{2}}{2 \beta\left(a^{2}-\left(\frac{\beta w}{2}\right)^{2}\right)^{2}} \\
I_{1}=\frac{4 a \frac{\beta w}{2}}{2 \beta\left(a^{2}-\left(\frac{\beta w}{2}\right)^{2}\right)^{2}} \\
I_{1}=\frac{a w}{\left(a^{2}-\left(\frac{\beta w}{2}\right)^{2}\right)^{2}}
\end{gathered}
$$

If $\beta=0$ 


$$
I_{1}=\frac{a w}{\left(a^{2}\right)^{2}}=\frac{w}{a^{3}}
$$

Substituting $a=z+\theta y+H / 2$

$$
I_{1}=\frac{w}{\left(z+\theta y-\frac{H}{2}\right)^{3}}
$$

The equation 3-28 correlates with the equation 3-18. Now,

$$
I_{1}=\frac{a w}{\left(a^{2}-\left(\frac{\beta w}{2}\right)^{2}\right)^{2}}
$$

Substituting $a=z+\theta y+H / 2$

$$
\begin{gathered}
I_{1}=\frac{w\left(z+\theta y-\frac{H}{2}\right)}{\left(\left(z+\theta y-\frac{H}{2}\right)^{2}-\left(\frac{\beta w}{2}\right)^{2}\right)^{2}} \\
I_{2}=\int_{-\frac{L}{2}}^{\frac{L}{2}} I_{1} d y
\end{gathered}
$$




$$
I_{2}=\int_{-\frac{L}{2}}^{\frac{L}{2}} \frac{w\left(z+\theta y-\frac{H}{2}\right)}{\left(\left(z+\theta y-\frac{H}{2}\right)^{2}-\left(\frac{\beta w}{2}\right)^{2}\right)^{2}} d y
$$

$$
I_{2}=\left[\frac{-w}{\left(2 \theta\left(\left(z+\theta y-\frac{H}{2}\right)^{2}-\left(\frac{\beta w}{2}\right)^{2}\right)\right)^{2}}\right]_{-\frac{L}{2}}^{\frac{L}{2}}
$$

Eqn 3-33

$$
I_{2}=\left[\frac{-w}{\left(2 \theta\left(\left(z+\theta\left(\frac{L}{2}\right)-\frac{H}{2}\right)^{2}-\left(\frac{\beta w}{2}\right)^{2}\right)\right)^{2}}\right]
$$

$$
-\left[\frac{-w}{\left(2 \theta\left(\left(z+\theta\left(\frac{-L}{2}\right)-\frac{H}{2}\right)^{2}-\left(\frac{\beta w}{2}\right)^{2}\right)\right)^{2}}\right]
$$

Eqn 3-34 


$$
\begin{gathered}
I_{2}=\frac{-w}{\left(2 \theta\left(\left(z+\frac{\theta L}{2}-\frac{H}{2}\right)^{2}-\left(\frac{\beta w}{2}\right)^{2}\right)\right)^{2}} \\
+\frac{w}{\left(2 \theta\left(\left(z-\frac{\theta L}{2}-\frac{H}{2}\right)^{2}-\left(\frac{\beta w}{2}\right)^{2}\right)\right)^{2}} \\
F_{i}=\frac{A}{6 \pi} \times I_{2}
\end{gathered}
$$

The above expression is the final equation for the integration of the smooth region of the slider. Similarly we found the moments of the slider about its $\mathrm{x}$ and $\mathrm{y}$ axis. As the second integrals of the moment equations were complicated, we performed only the first iteration of the integration for each of the moments. The moment about the x-axis, $M_{v y}$ is given by,

$$
M_{v y}=\frac{A}{6 \pi} \int_{\frac{-w}{2}}^{\frac{w}{2}} \int_{\frac{-L}{2}}^{\frac{L}{2}} \frac{x d x d y}{h^{3}}
$$

First integrating this with respect to $x$

$$
I_{3}=\int_{\frac{-w}{2}}^{\frac{w}{2}} \frac{x d x}{\left(z+\theta y-\beta x-\frac{H}{2}\right)^{3}}
$$




$$
I_{3}=\int_{\frac{-w}{2}}^{\frac{w}{2}} \frac{d x}{\left(-\beta x+\left(z+\theta y-\frac{H}{2}\right)\right)^{3}}
$$

Let $a=z+\theta y+H / 2, b=-\beta$

The above equation is in the form of

$$
\begin{gathered}
\int \frac{x d x}{(a+b x)^{3}}=\frac{-1}{2 b} \int x d\left[\frac{1}{(b x+a)^{2}}\right] \\
=-\frac{1}{2 b} \frac{x}{(a+b x)^{2}}+\frac{1}{2 b} \int \frac{d x}{(a+b x)^{2}}+c \\
\int \frac{x d x}{(a+b x)^{3}}=-\frac{1}{2 b} \frac{x}{(a+b x)^{2}}-\frac{1}{2 b} \frac{(a+b x)}{(a+b x)^{2}}+c \\
=-\frac{1}{2 b^{2}} \frac{b x}{(a+b x)^{2}}-\frac{1}{2 b^{2}} \frac{(a+b x)}{(a+b x)^{2}} \\
=-\frac{(a+2 b x)}{2 b^{2}(a+b x)^{2}}
\end{gathered}
$$

Substituting $b=-\beta$ 


$$
\begin{gathered}
I_{3}=\left[\frac{(2 \beta x-a)}{2 \beta^{2}(a-\beta x)^{2}}\right]_{-\frac{w}{2}}^{\frac{w}{2}} \\
I_{3}=\left[\frac{\left(2 \beta\left(\frac{w}{2}\right)-a\right)}{2 \beta^{2}\left(a-\beta\left(\frac{w}{2}\right)\right)^{2}}-\frac{\left(2 \beta\left(\frac{-w}{2}\right)-a\right)}{2 \beta^{2}\left(a-\beta\left(\frac{-w}{2}\right)\right)^{2}}\right] \\
I_{3}=\frac{w}{2 \beta}\left[\frac{(\beta w-a)}{2 \beta^{2}\left(a-\frac{\beta w}{2}\right)^{2}}+\frac{(\beta w+a)}{2 \beta^{2}\left(a+\frac{\beta w}{2}\right)^{2}}-\frac{\left(a-\frac{\beta w}{2}\right)^{2}}{2 \beta^{2}\left(a-\frac{\beta w}{2}\right)^{2}}\right. \\
-\frac{\left.a+\frac{\beta w}{2}\right)^{2}}{2 \beta^{2}\left(a+\frac{\beta w}{2}\right)^{2}} \\
\left.\frac{-\frac{\beta}{2 \beta^{2}}\left[\frac{1}{\left(a-\frac{\beta w}{2}\right)^{2}}-\frac{1}{\left(a+\frac{\beta w}{2}\right)^{2}}\right]}{2}\right]
\end{gathered}
$$

Eqn 3-49 


$$
\begin{aligned}
I_{3}=\frac{w}{2 \beta}\left[\frac{\left(a+\frac{\beta w}{2}\right)^{2}+\left(a-\frac{\beta w}{2}\right)^{2}}{\left.\left(a-\frac{\beta w}{2}\right)^{2}\left(a+\frac{\beta w}{2}\right)^{2}\right]}\right. \\
\\
\quad-\frac{a}{2 \beta^{2}}\left[\frac{\left(a+\frac{\beta w}{2}\right)^{2}-\left(a-\frac{\beta w}{2}\right)^{2}}{\left(a-\frac{\beta w}{2}\right)^{2}\left(a+\frac{\beta w}{2}\right)^{2}}\right]
\end{aligned}
$$

Eqn 3-50

$$
I_{3}=\frac{w}{2 \beta}\left[\frac{2\left(a^{2}+\left(\frac{\beta w}{2}\right)^{2}\right)}{\left(a^{2}-\left(\frac{\beta w}{2}\right)^{2}\right)^{2}}\right]-\frac{a}{2 \beta^{2}}\left[\frac{2 \beta a w}{\left(a^{2}-\left(\frac{\beta w}{2}\right)^{2}\right)^{2}}\right]
$$

Eqn 3-51

$$
I_{3}=\frac{w}{\beta}\left[\frac{a^{2}+\left(\frac{\beta w}{2}\right)^{2}}{\left(a^{2}-\left(\frac{\beta w}{2}\right)^{2}\right)^{2}}\right]-\frac{a^{2} w}{\beta}\left[\frac{1}{\left(a^{2}-\left(\frac{\beta w}{2}\right)^{2}\right)^{2}}\right]
$$

Eqn 3-52

$$
I_{3}=\frac{\beta w^{3}}{4\left(a^{2}-\left(\frac{\beta w}{2}\right)^{2}\right)^{2}}
$$

Substituting $a=z+\theta y+H / 2$ 


$$
I_{3}=\frac{\beta w^{3}}{4\left(\left(z+\theta y-\frac{H}{2}\right)^{2}-\left(\frac{\beta w}{2}\right)^{2}\right)^{2}}
$$

$$
M_{v y}=\int_{-\frac{L}{2}}^{\frac{L}{2}} I_{3} d y
$$

The equation 3-55 is then evaluated using a MATLAB function trapz, which integrates the equation numerically using trapezoidal method. Similarly the moment about y-axis, $M_{v x}$, is evaluated as follows.

$$
M_{v x}=\frac{A}{6 \pi} \int_{\frac{-w}{2}}^{\frac{w}{2}} \int_{\frac{-L}{2}}^{\frac{L}{2}} \frac{y d x d y}{h^{3}}
$$

First integrating this with respect to $x$

$$
I_{4}=y \int_{\frac{-w}{2}}^{\frac{w}{2}} \int_{\frac{-L}{2}}^{\frac{L}{2}} \frac{d x d y}{h^{3}}
$$

The above equation is similar to the equation 3-14, therefore, by simplifying we get 


$$
I_{4}=\int_{-L / 2}^{L / 2} y\left(\frac{w\left(z+\theta y-\frac{H}{2}\right)}{\left(\left(z+\theta y-\frac{H}{2}\right)^{2}-\left(\frac{\beta w}{2}\right)^{2}\right)^{2}}\right) d y \quad \text { Eqn 3-58 }
$$

The above equation is also using the MATLAB function trapz, to get the moments. The results obtained from these equations were then added to the results obtained in the five-degree-of-freedom model and case studies were performed.

\subsection{Theory on thermally expanding stationary bump}

The concept of thermally expanding bump was discovered by chance. The $\mathrm{read} / \mathrm{write}$ element in the slider have coils which are used to magnetize the magnetic layer of the disk so as to store data. When this elements was supplied current, the read/write element expanded, making the element to expand towards the disk resulting to a reduced flying height with the disk wear and slider wear. To counter this, researchers found a way to solve this problem. They increased the flying height of the slider and introduced a heater element in the slider manufacturing which was used to control the flying height while the $\mathrm{read} / \mathrm{write}$ function was being performed.

The theory for a thermally expanding bump can be explained using the figure 3.6. The idealized 1D plot shows a heater element attached to a cantilever beam hanging down. A metal bar with insulated sides is attached to the heater. The temperature gradient from the heater to its ends is shown in the plot. This setup is held over a disk revolving with a velocity $V_{d}$. 


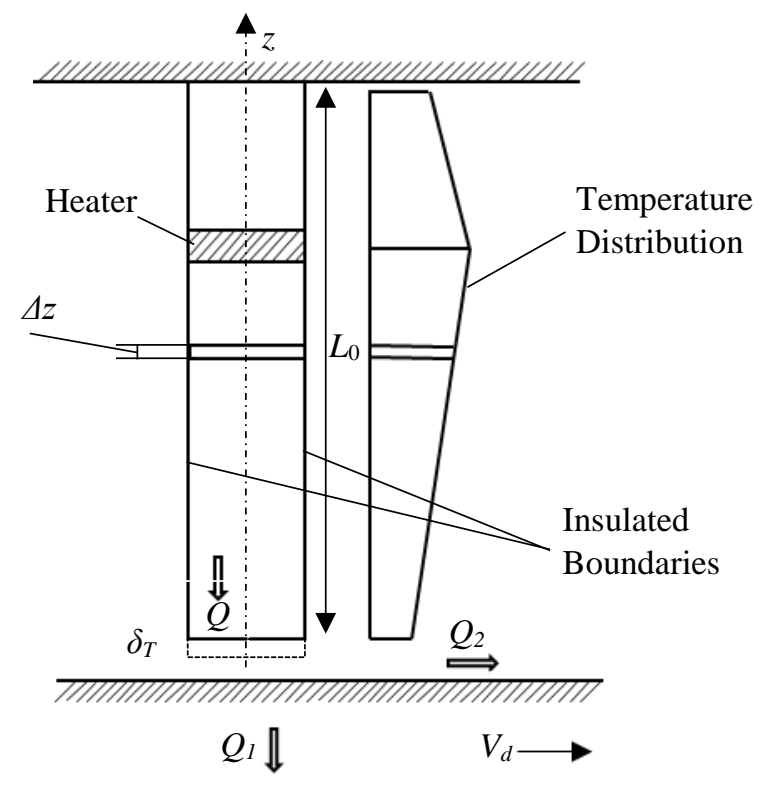

Figure 3.6 The theory behind the working of thermally expanding bump (Onedimensional model)

When the heater is switched on, it will expand and due to conduction in the bar. Let $\mathrm{Q}$ be the amount of heat transferred into the element when the heater is turned on, $T_{0}$ be the initial temperature of the bar element. When the heater is switched on, the total heat generated is distributed towards the bar element. As the walls of the bar is insulated, the heat will be directed down towards the disk. The heat generated is divided into two components. $\mathrm{Q}_{1}$ is the heat transferred to the disk due to conduction. $\mathrm{Q}_{2}$ is the heat transferred into the air, by convection, due to the air stream. Let us consider a small element of width $\Delta y$ within the bar. The thermal expansion in this element is given as

$$
d \delta_{T}=C_{T}\left(T-T_{0}\right) d y
$$


where $T$ is the temperature of the element after the heater is switched on, $C_{T}$ is the constant of expansion, $\mathrm{K}^{-1}$ or ${ }^{\circ} \mathrm{C}^{-1}$. The total thermal expansion in the bar is given by

$$
\begin{array}{cc}
\delta_{T}=\int_{0}^{L_{0}} d \delta_{T}=\int_{0}^{L_{0}} C_{T}\left(T-T_{0}\right) d y & \text { Eqn 3-60 } \\
\delta_{T}=C_{T} \int_{0}^{L_{0}}\left(T-T_{0}\right) d y & \text { Eqn 3-61 }
\end{array}
$$

The above expression is basic principle behind which this technology works. Due to very small value of $C_{T}$ (because of the dimensions of the read/write element of the slider), the above principle considered as the theory for nano-scale thermal actuation.

The coils are magnetized by the read/write D.C current. Due to magnetization, heat is generated in the element. This is known as the joule heating. The power generated by the heater is given by

$$
P_{h}=V \times I=I^{2} \times R \quad \text { Eqn 3-62 }
$$

here $P_{h}$ is the heater power, $V$ is the voltage supplied to the heater, $I$ is the current supplied to the heater, and $R$ is the resistance offered by the heating element. The emphasis of this the thermally actuated flying-height control is to control the heater element, which will help us to control the gap in the hard disk drive.

In this research, a function was formulated to simulate the thermally expanding bump from the slider surface towards the disk. Two time constants $\tau_{1}$ and $\tau_{2}$ were used to 
control the heater temperature, where $\tau_{1}$ is the time constant for heating and $\tau_{2}$ is the time constant for the cooling process [24]. The heater was modelled such that if the heater was not turned off, then the bump would continue to expand and would approach the steady state height $h_{s s}$ as the time approaches infinity. The $\tau_{1}$ and $\tau_{2}$ were set such that, the heater would turn off when the bump height of $63 \%$ of the $h_{s s}$ was achieved and the cooling was allowed until the height of the bump was $63 \%$ smaller than the original heated bump. The controlling of the bump is shown in the figure 3.7 .

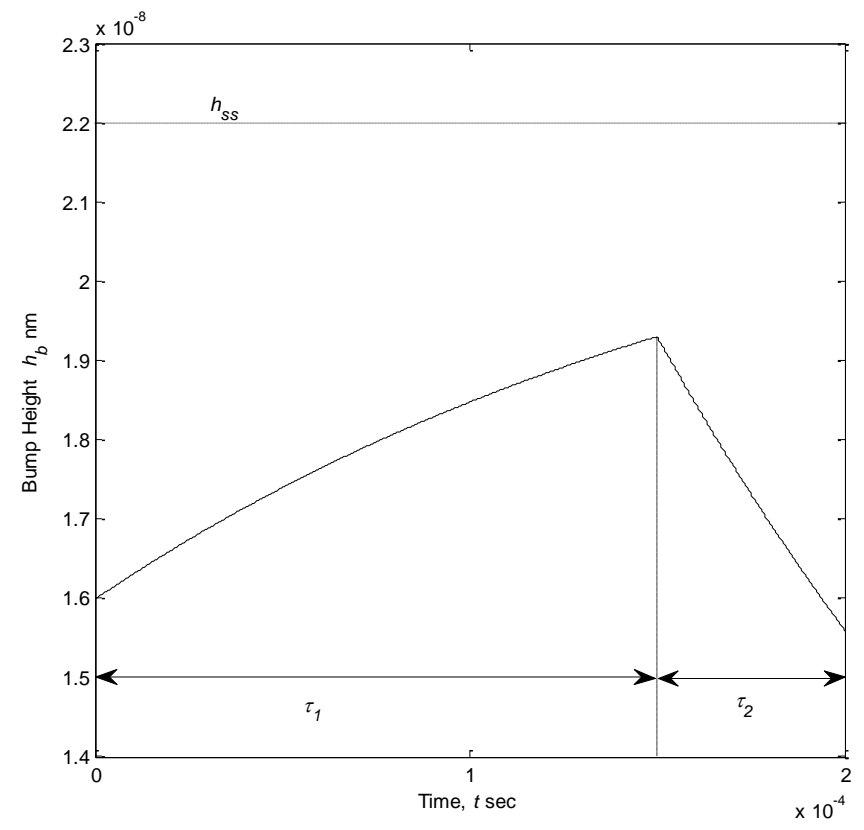

Figure 3.7 The heater function curve

\subsection{Theory on non-linear spring approach}

So far the research has been on an assumption that the spring system is linear. But to meet the real world application, the spring needs to be non-linear. For cases with larger 
interferences or for the stationary thermally expanding stationary bump, a non-linear spring model has been used. To make the springs non-linear, we need to understand the contact mechanics of the system.

In the research we have assumed that the bump on the disk is stiff and it completely displaces when the slider comes in contact with it. But in reality the bump acts as a rubber ball, which when comes in contact at a surface, only the surface of contact gets displaced and the rest of the ball displaces accordingly. This can be explained by the Hertzian contact model.

\section{Hertzian Contact Model}

Hertz theory of contact tells us about the contact between two non-adhesive bodies and their behavior when they are under tension [27]. It says that the two bodies in contact can be separated without adhesion forces. The compressing load is related to the pressure by,

$$
\begin{gathered}
P=\int_{0}^{a} p(r) 2 \pi d r \\
P=\frac{2}{3} p_{0} \pi a^{2}
\end{gathered}
$$

Where, 


$$
\begin{gathered}
a=\frac{\pi p_{0} R}{2 E^{*}}=\left(\frac{3 P R}{4 E^{*}}\right)^{\frac{1}{3}} \\
\delta=\frac{a^{2}}{R}=\left(\frac{9 P^{2}}{16 R E^{* 2}}\right)^{\frac{1}{3}} \\
p_{0}=\frac{3 P}{2 \pi a^{2}}=\left(\frac{6 P E^{* 2}}{\pi^{3} R^{2}}\right)^{1 / 3}
\end{gathered}
$$

Here, $a$ is the area of contact, $E^{*}$ is the combined elastic moduli, $R$ is the relative radius of curvature, $\delta$ is the displacement after contact, and $p_{0}$ is the maximum pressure applied $[27,28]$.

The assumptions of the Hertz theory are as follows:

- The surfaces are continuous and non-conforming

- The strains are small

- The surfaces are frictionless

- Each solid is considered as an elastic half space.

From the above assumptions we can conclude that the Hertz contact theory can be applied to our research when we don't consider the effects of the Intermolecular forces. But when the Intermolecular forces are considered then we need to take adhesion into consideration. There are majorly two well-known models. They are JKR model and DMT model. 
JKR (Johnson-Kendell-Roberts) Model

It was proposed by K.L Johnson, K. Kendell and A.D Roberts in 1971 [31] which assumes the adhesion in elastic bodies in contact. It considers the pressure of adhesive forces only inside the area of contact. It considers the balance between the stored elastic energy and loss in surface energy.

The pressure distribution in the contact region is given by

$$
p(r)=p_{0}\left(1-\frac{r^{2}}{a^{2}}\right)^{\frac{1}{2}}+p^{\prime}{ }_{0}\left(1-\frac{r^{2}}{a^{2}}\right)^{-\frac{1}{2}}
$$

where

$$
p_{0}=\frac{2 a E^{*}}{\pi R} ; p_{0}^{\prime}=-\left(\frac{4 \gamma E^{*}}{\pi a}\right)^{\frac{1}{2}}
$$

The displacement

$$
\delta=\left(\frac{\pi a}{2 E^{*}}\right)\left(p_{0}+2 p_{0}^{\prime}\right)
$$

The net contact force is 


$$
P=\int_{0}^{a} 2 \pi r p(r) d r=\left(\frac{2}{3} p_{0}+2 p^{\prime}{ }_{0}\right) \pi a^{2}
$$

$\mathrm{P}$ and a are related by

$$
\left(P-\frac{4 E^{*} a^{3}}{3 R}\right)^{2}=16 \pi \gamma E^{*} a^{3}
$$

where $\gamma$ is the surface energy per unit area of each surface, $p^{\prime}{ }_{0}$ is the pull-off force. The above relation can be described by the figure 3.8 .

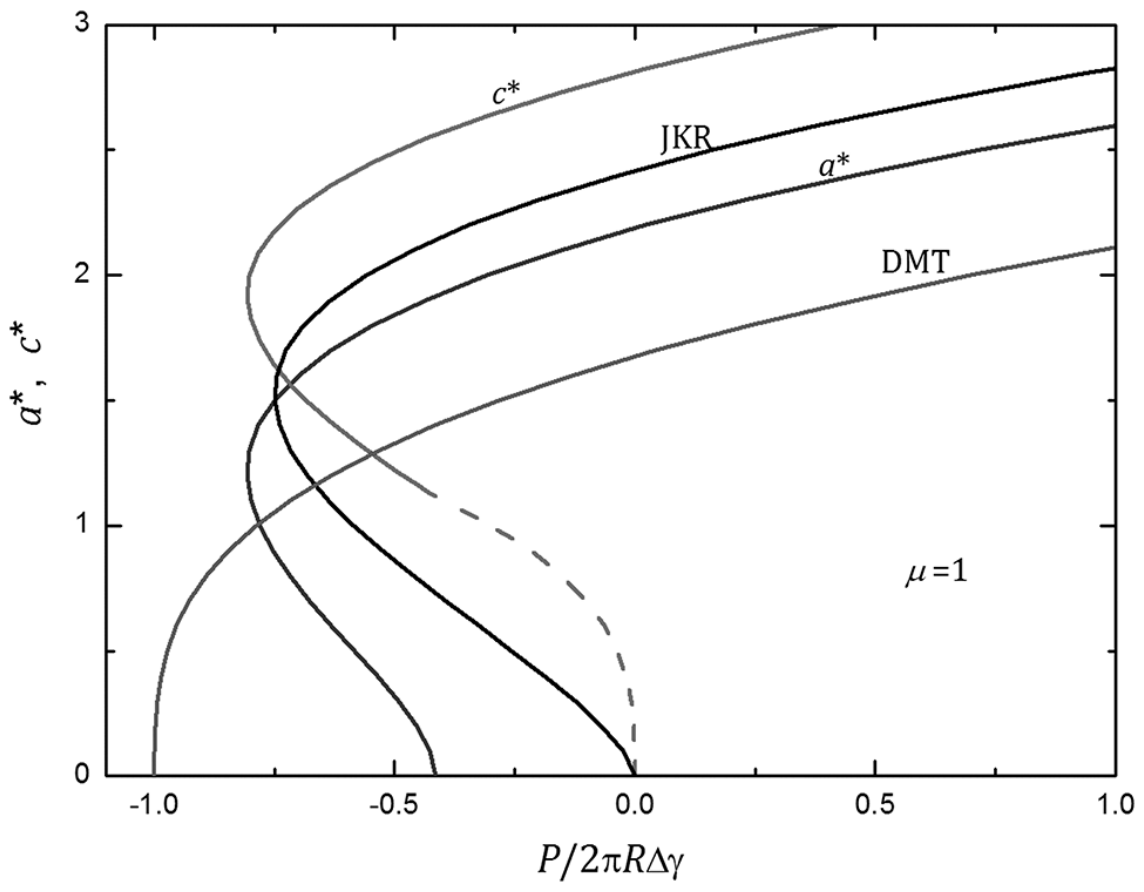

Figure 3.8 Different curves for contact theories 
DMT (Derjaguin-Muller-Toporov) Model

It was proposed by B.V Derjaguin, V.M Muller and YU.P Toporov in 1974. This model considers the surface of contact remains the same as in Hertzian contact but there is an additional attractive interactions outside the area of contact [32]. Their theory opposed the JKR theory of adhesive contact mechanics. They formulated their theory as follows.

The force of adhesion between two rigid surfaces R1 and R2, is given by the Bradley theory as

$$
P_{A}=2 \pi \Delta \gamma R
$$

The contact radius proposed by DMT model is given by

$$
a^{3}=\frac{3 R}{4 E^{*}}(P+2 \pi \Delta \gamma R)
$$

The pull-off force is given by

$$
p^{\prime}{ }_{0}=-2 \pi \Delta \gamma R
$$

here $\Delta \gamma$ is the equivalent surface energy of the bodies in contact.

Zhao and Shi [29] compared JKR and DMT models with the Hertz theory with respect to the nano-contact problems and proved that under small loads, the contact radius 
was dominated by the work of adhesion and indenter tip radius $\mathrm{R}$. When the loads were large, the adhesion was dominated by the strain energy of deformation. They concluded that JKR was valid for low modulus, high surface energy materials, large indenters and DMT was valid for highly rigid surfaces, low surface energy materials and small indenters.

As both the theories try to show the same phenomenon. To decide which theory is apt, D. Tabor in 1977 showed that both the theories (JKR and DMT) where extreme cases of study [33] and we could decide which theory could be used using a Tabor coefficient [30] defined as

$$
\mu_{\text {Tabor }} \equiv\left(\frac{R \Delta \gamma^{2}}{E^{*^{2}} \varepsilon^{3}}\right)^{\frac{1}{3}}
$$

where $\mu_{\text {Tabor }}$ is the Tabors coefficient, $\varepsilon$ is the separation between the two bodies. If the tabors coefficient was as small as 0.2 , Greenwood suggested to use DMT and if it was above that of 3.0, then JKR was to be used [30,31].

In our research, as adhesion was taken into consideration, we used the Tabors coefficient to decide model of study. When we put in the parameters, we conclude that we need to use the JKR model in our case. The figure 3.9 shows the confirmation that our model follows the JKR model. The preliminary results for the JKR model were obtained in preparation for the further expansion of the model, but the current MATLAB program is based on the linear and nonlinear spring formulations. 


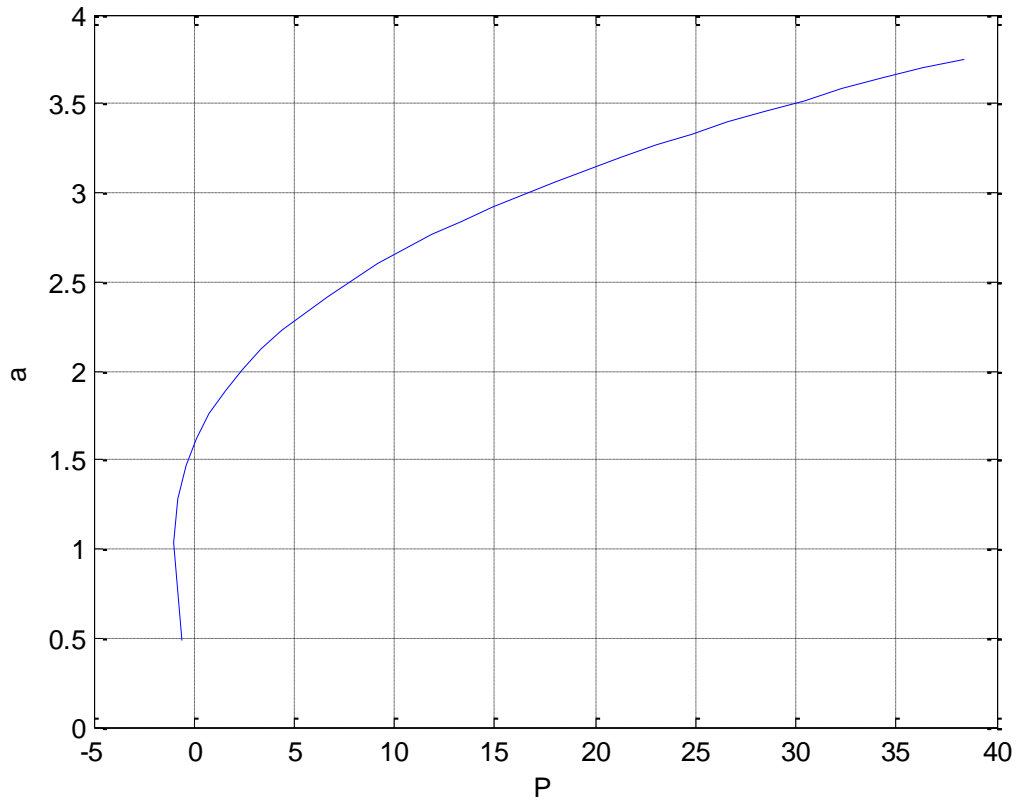

Figure 3.9 Validation of JKR nature of the research 


\section{CHAPTER IV}

\section{RESULTS AND DISCUSSION}

\section{Skew Definition}

Skew is the circumfrancial direction of the slider which helps in the determining the location of the slider with respect to the disk. The figure 4.1 will help us understand its meaning.

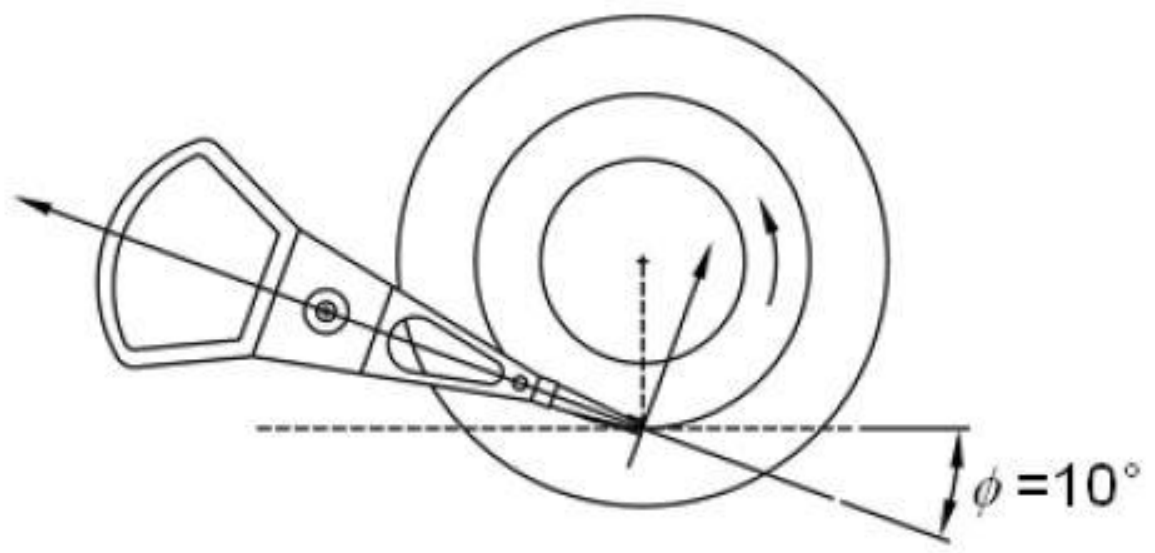

Figure 4.1 Skew Definition [34]

In this study, we have used a simplified five-degree-of-freedom slider arm assembly which has slider dimensions (length $\times$ width $\times$ thickness) as $1.25 \times 1 \times 0.3 \mathrm{~mm}^{3}$, a slider mass of $1.58 \mathrm{mg}$, a trailing edge flying height of $10 \mathrm{~nm}$. The disk stiffness $\mathrm{k}_{\mathrm{d}}$ and damping coefficients used are $1.5 \times 10^{6} \mathrm{~N} / \mathrm{m}$ and $0.3079 \mathrm{~N}-\mathrm{s} / \mathrm{m}$, respectively. The pitch angle was initially set to $190 \mu \mathrm{rad}$, and actuator arm stiffness $\mathrm{k}_{\mathrm{arm}}=216 \mathrm{~N} / \mathrm{m}[11,34]$. The 
Hamaker Constants A and B used for the intermolecular studies are $7.0191 \times 10^{-19}$ and $7.0191 \times 10^{-76}$ (see at APPENDIX C for the calculation), respectively.

Many cases have been considered to understand the behavior of the slider under the influence of the intermolecular forces and thermally expanding bumps. We started our analysis by analyzing the case with a single paraboloidal bump to track the slider vibrations under the influence of intermolecular forces. The radius of the bump $r_{b}$ is assumed to be 15 $\mu \mathrm{m}$, the height of the bump $\mathrm{h}_{\mathrm{b}}$ to be $20 \mathrm{~nm}$ and skew angle of the slider with respect to that to the circumferential direction to be $10^{\circ}$.
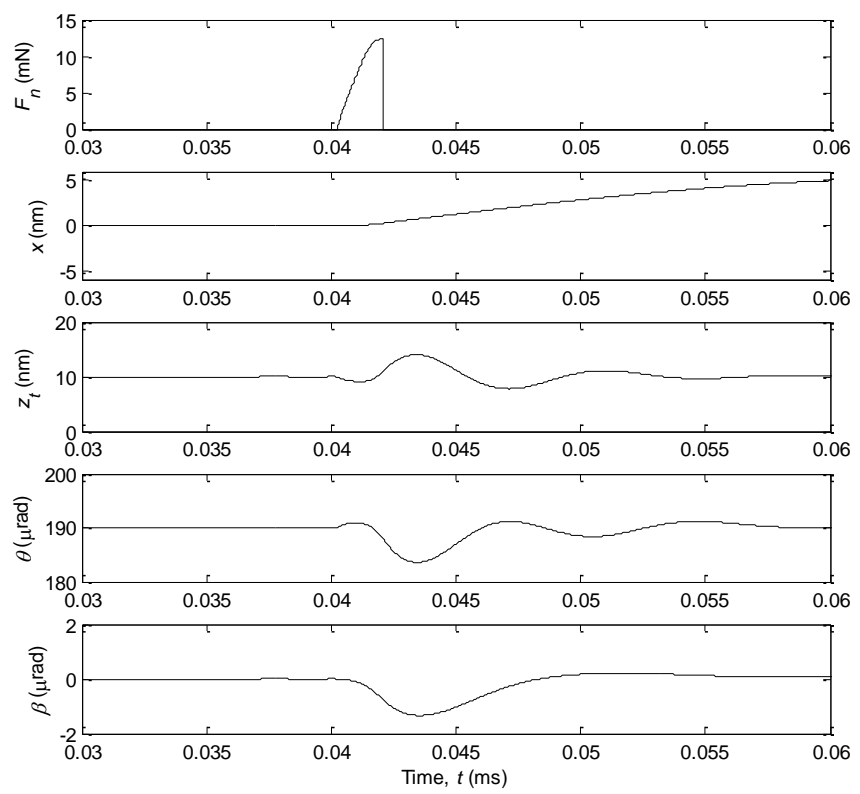

Figure 4.2 Response of the slider to single bump with a height of $20 \mathrm{~nm}$, a bump radius of

$$
15 \mu \mathrm{m} \text { and a skew, } \phi=10^{\circ}
$$

From the given conditions, we clearly know that there would a contact as the height of the bump is more than that of the flying height, but we observe (see figure 4.2) downward movement of the slider before the contact $(\mathrm{t}<0.04 \mathrm{~ms})$. This occurs due to the 
presence of the attractive intermolecular forces, and these forces result in increased (just after $0.04 \mathrm{~ms}$ ) normal contact forces, and transverse vibrations of the slider, evident form the $x$-displacement plot.

\section{Effects of the Bump Height and Bump Radius}

We observe the response of the slider in different conditions which will support the fact that the intermolecular forces contribute in increased transverse vibrations and normal contact forces. First, by changing the height of the bumps in both the cases, we have observed the following:

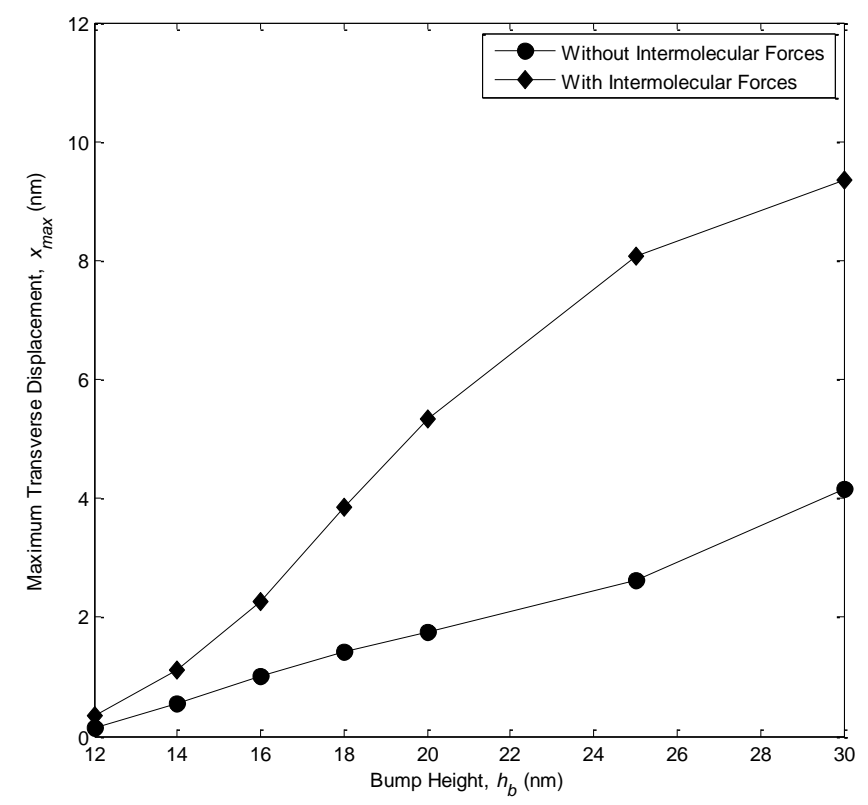

Figure 4.3 Maximum transverse displacement, $x_{\max }$,vs. the bump height $h_{b}$

When intermolecular forces are not considered, we see that the $x_{\max }$ increases gradually as the height of the bump increases (see figure 4.3). As the height of the bump increases, the area of the bump coming in contact with the slider increases and hence the 
contact forces will increase. This will result in the increasing transverse displacement $x$ with respect to increasing height of the bump. We observe the similar trend when intermolecular forces are considered, but we see larger displacements when compared to the previous case because the intermolecular forces tend to pull the slider down, resulting to increase in the contact forces, and this will result in more transverse displacements.

Similarly, as we compare the normal contact forces with the bump height, we see that the normal contact force increases with the increasing bump height when intermolecular forces are not considered as shown in the figure 4.4.

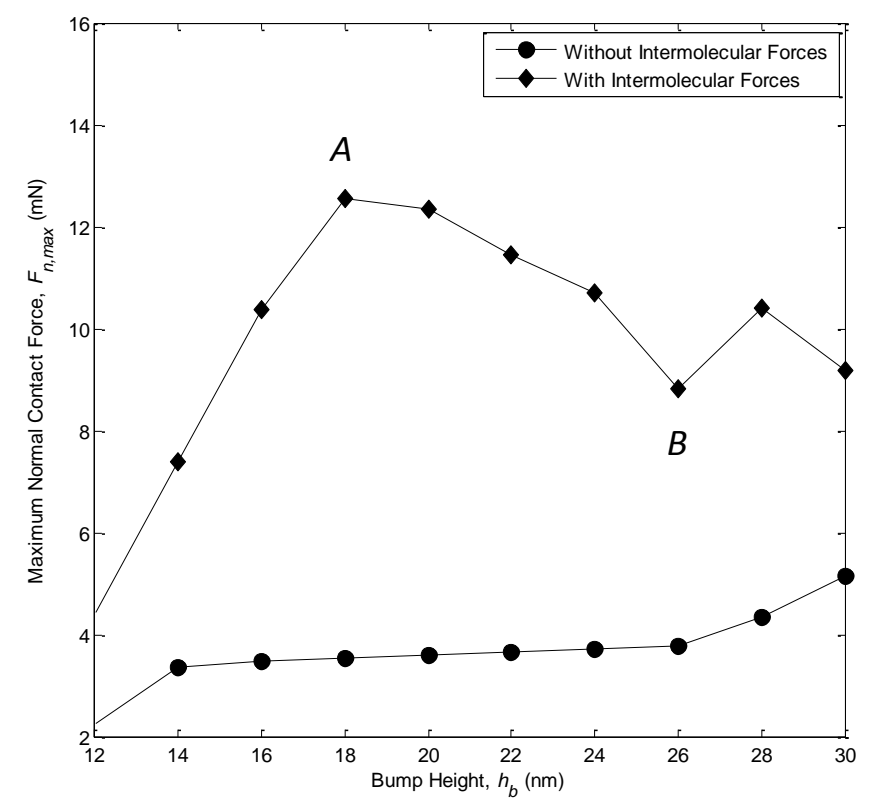

Figure 4.4 Maximum normal contact force $F_{n, \max }$ vs. the bump height $h_{b}$

In contrast, when the intermolecular forces are considered, we initially see that the normal contact forces increase with the increasing bump height until point $A(18 \mathrm{~nm})$ and, then, starts to decrease, with a magnitude still above curve for the cases without intermolecular forces, gradually until point $B(26 \mathrm{~nm})$ and, then, starts to increase again. 
Examining the raw data graphs (see APPENDIX D) for different bump heights, it has been found that there is a transition at the bump height at point $\mathrm{A}$ for both the curve of the pitch angle, $\theta$, and the curve for the trailing edge height $z_{t}$. For $h_{b}<18 \mathrm{~nm}$, there is a significant increase of the pitch angle, $\theta$, and a significant decrease of the trailing-edge flying height, $z_{t}$, before other smaller oscillations.

These two phenomena can be attributed to the fact that the attractive intermolecular forces pull the slider toward the bump on the disk, noting that the moving bump reaches a position close to the trailing edge of the slider when these interactions occur. This slight downward motion of the slider reduces the spacing, thus increasing slightly the magnitude of the attractive intermolecular forces, which partially act as an additional load on the contact interface and, thus, slightly increase the contact force above the level of a situation with the slider at the initial equilibrium position.

When the bump height, $h_{b}$, exceeds $18 \mathrm{~nm}$, both the increase in the pitch angle, $\theta$, and the decrease in the trailing-edge flying height, $z$, induced by the intermolecular forces become smaller while the collision effect, opposite to the above intermolecular effect, becomes dominant. The collision, also observed in the solutions without considering intermolecular forces, occurs at a location on the slider close to its trailing edge, exhibiting a large decrease of the pitch angle, $\theta$, and a large increase of the trailing-edge flying height, $z_{t}$. In the time sequence as observed from the curves of the pitch angle, $\theta$, and the trailingedge flying height, $z$, this collision mechanism occurs just after that of the weakened intermolecular force effects. Because the slider moves away from the disk compared to the initial equilibrium position due to the collision, there is a net decrease of the portion of the contact force that is caused by the attractive intermolecular forces (as additional contact 
loading). Therefore, after exceeding some particular bump height $(18 \mathrm{~nm})$, the contact force, $F_{n}$, decreases, with the intermolecular forces considered. From the above observations, the increase-and-then-decrease trend of the curve for the normal contact force, $F_{n}$, versus bump height, $h_{b}$, for the cases with consideration of the intermolecular forces can be attributed to the change of the contact behavior from an intermolecular forcedominated regime for bump height $h_{b}<18 \mathrm{~nm}$ to a collision-dominated regime for $h_{b}>18$ nm.

When the height of the bump, $h_{b}$, is set to $18 \mathrm{~nm}$, we study two cases in which we observe the Maximum transverse displacement and the maximum normal contact forces with respect to varying radius of the bump. The figure 4.5 shows the variation of maximum transverse displacement with the radius of the bump.

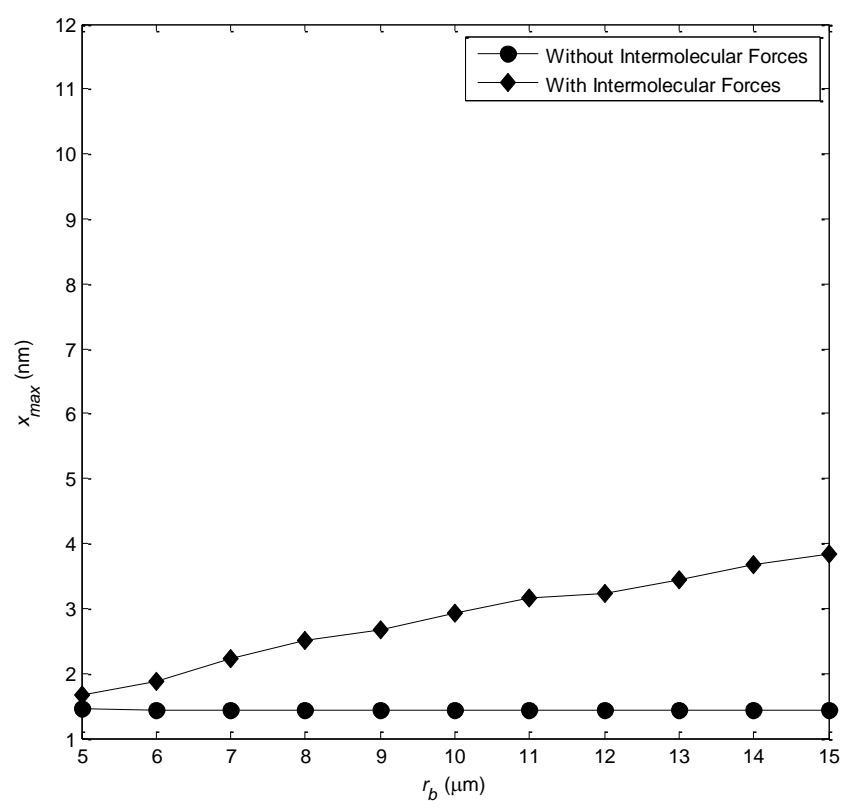

Figure 4.5 Maximum transverse displacement $x_{\max }$ vs the radius of the bump $r_{b}$ 
When the intermolecular forces are not considered, we see that the $x_{\max }$ remains the same for every radius of the bump. This is because the height of the bump is constant and the area of contact will not affect the contact as the bump is paraboloid, which was idealized as a spring earlier. But when the intermolecular forces are considered, the $x_{\max }$ increase with increasing bump radius. As the effective surface area of contact increases, the intermolecular forces per surface area increases (shown in figure 4.6), which will result in more transverse displacement. This is also the same reason for the normal contact forces to increase with the radius of the bump, as shown in the figure 4.7.

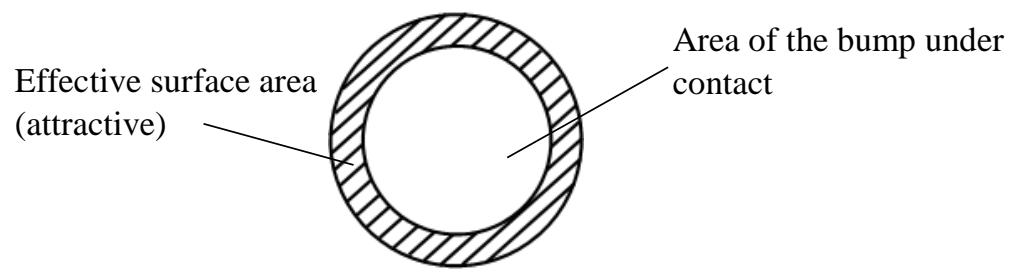

Figure 4.6 The contact regime for a paraboloidal bump

To justify the above theory, let us consider a case with height of the bump $h_{b}=12.5$ nm with a radius of bump $r_{b}=15 \mu \mathrm{m}$, which will give the following responses for both the conditions. 


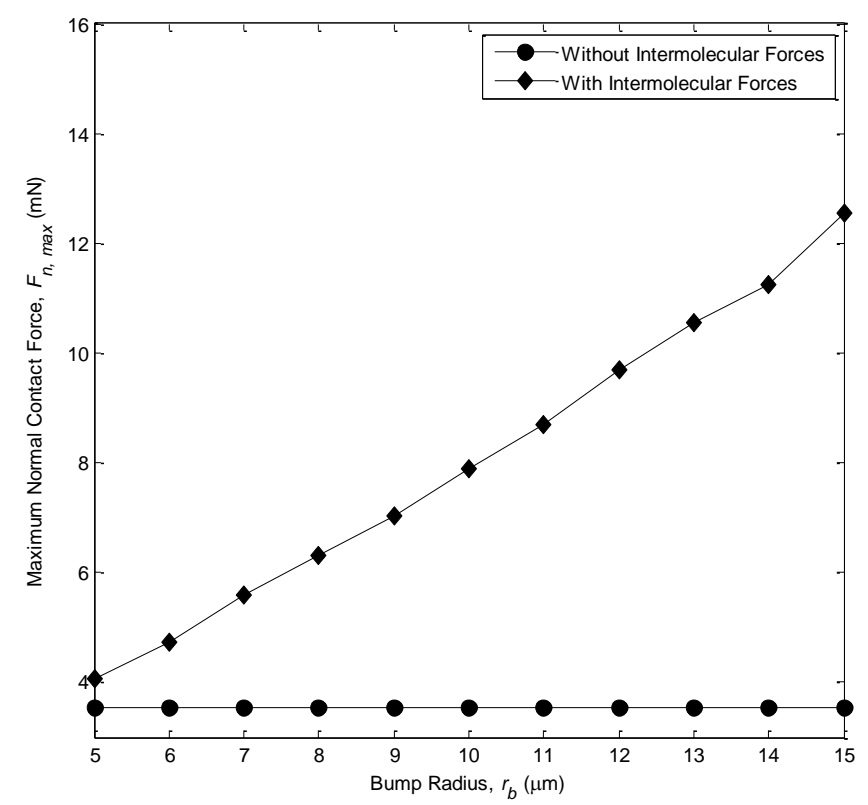

Figure 4.7 Maximum normal contact force $F_{n, \max }$ vs radius of the bump $r_{b}$

To justify the above theory, let us consider a case with height of the bump $h_{b}=12.5$ $\mathrm{nm}$ with a radius of bump $r_{b}=15 \mu \mathrm{m}$, which will give the following responses for both the conditions shown in figure 4.8 .

From the figure 4.8, we can see the variation in the response of the slider for different conditions. The response with intermolecular forces has a larger normal contact forces, $F_{n}$, when compared to the case without intermolecular forces. We also see that the transverse displacement, $x$, is larger in the condition where the intermolecular forces are considered. The third plot for the trailing-edge flying height, $z_{t}$, indicates the existence of the intermolecular forces as the slider moves down first slightly before the contact occurs. 

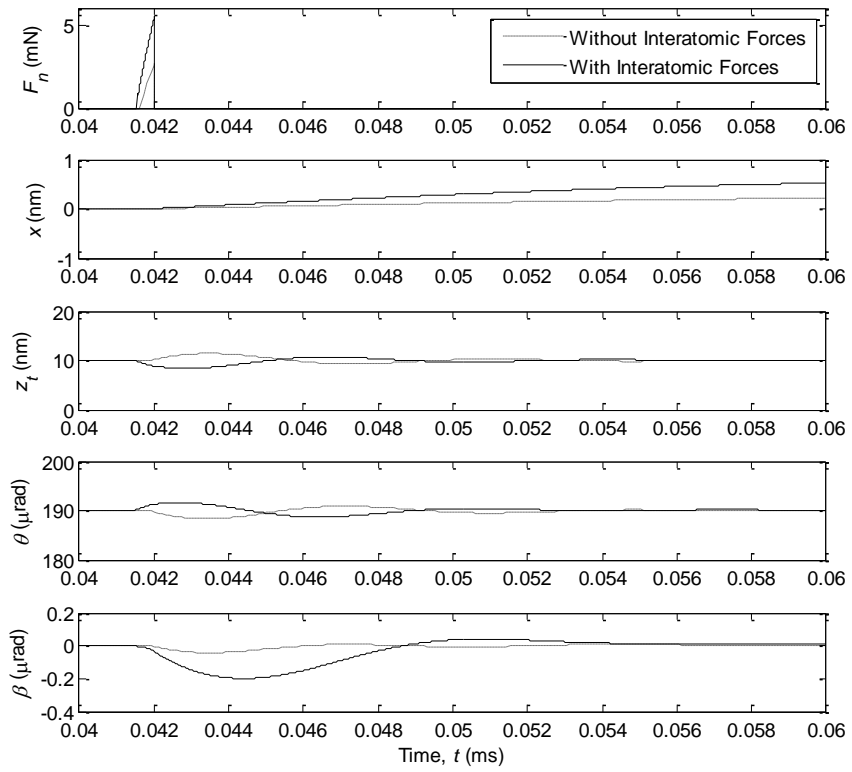

Figure 4.8 Response for the slider for $h_{b}=12.5 \mathrm{~nm}, r_{b}=15 \mu \mathrm{m}$ and skew $\phi=10^{\circ}$

\section{Effects of Skew and Coefficient of Friction}

The figure 4.9 shows the effect of skew on the maximum transverse displacement $x_{\max }$ in the cases with intermolecular forces and without intermolecular forces. We can see that the $x_{\max }$ increases linearly with the increase in the skew angle $\phi$. The figure 4.10 shows the response curve for $20^{\circ}$ skew $\phi$, when both intermolecular forces are considered and neglected respectively. 


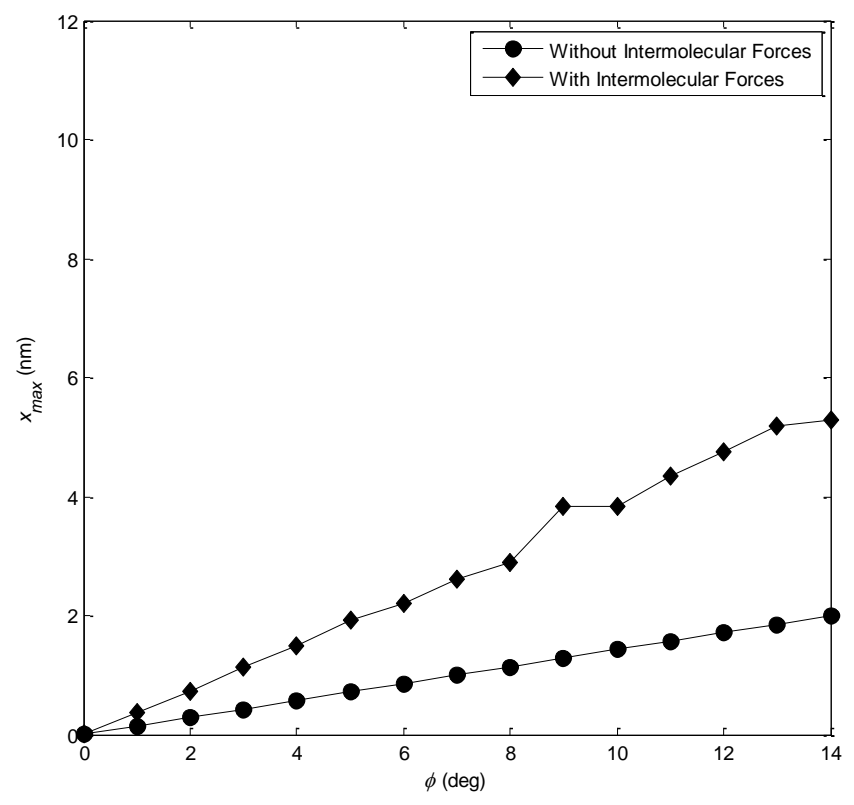

Figure 4.9 Maximum transverse displacement $x_{\max }$ vs. the skew angle $\phi$

Due to the big skew, the contact of the bump occurs at the edge of the slider, resulting to wobbling of the slider, which can be seen as the deviation in roll angle $\beta$ (see figure 4.10). There is more deviation in roll angle $\beta$, when the intermolecular forces are considered when compared to the case with no intermolecular forces. Similarly when the coefficient of friction is changed, we see a linear relationship between the maximum transverse displacement $x_{\max }$, when both the cases are compared as shown in the figure 4.11. 

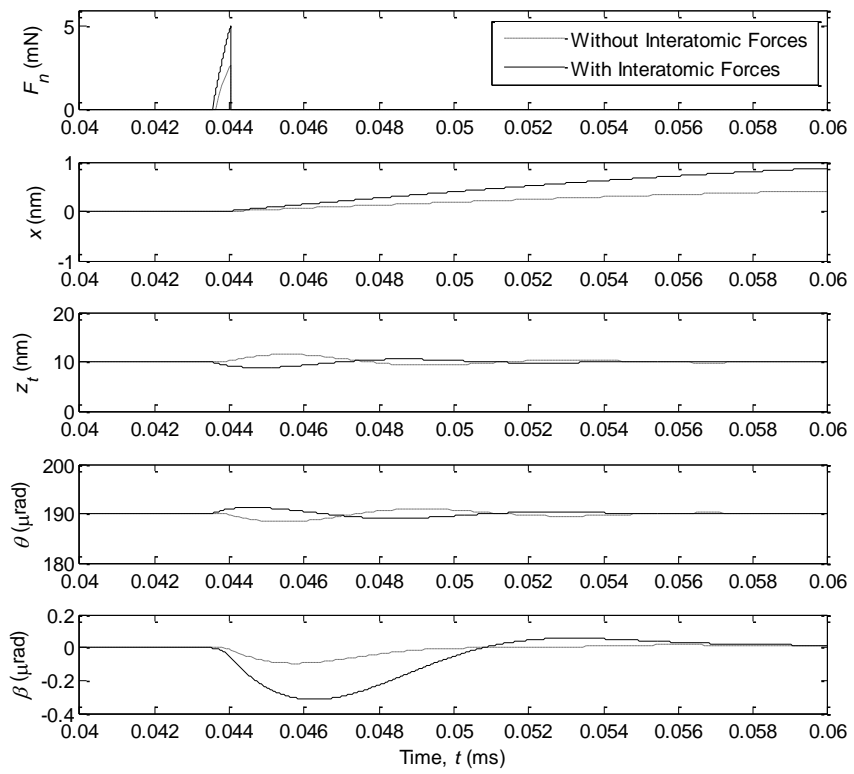

Figure 4.10 Response Comparison for $20^{\circ}$ skew with and without Intermolecular forces

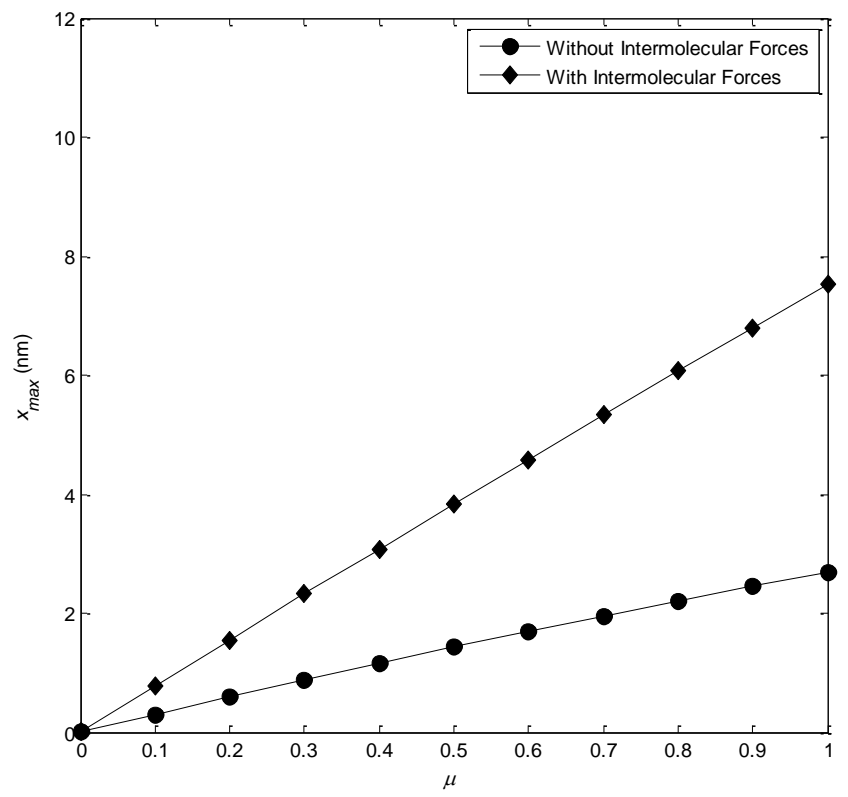

Figure 4.11 Maximum transverse displacement $x_{\max }$ vs the coefficient of friction $\mu$ 


\section{$\underline{\text { Single Bumps vs. Multiple lined bumps }}$}

When 6 bumps, which are radially aligned on the radial line, come in contact with the slider, we see aggravated results when compared to a single bump when we decouple the roll angle $\beta$ with zero skew. This is because the contact force is six times the original case and this will result in amplified results. Decoupling is done so that we have uniform force distribution in the bumps. This is done by artificially setting the stiffness to zero. The comparison responses are given the figure 4.12 .
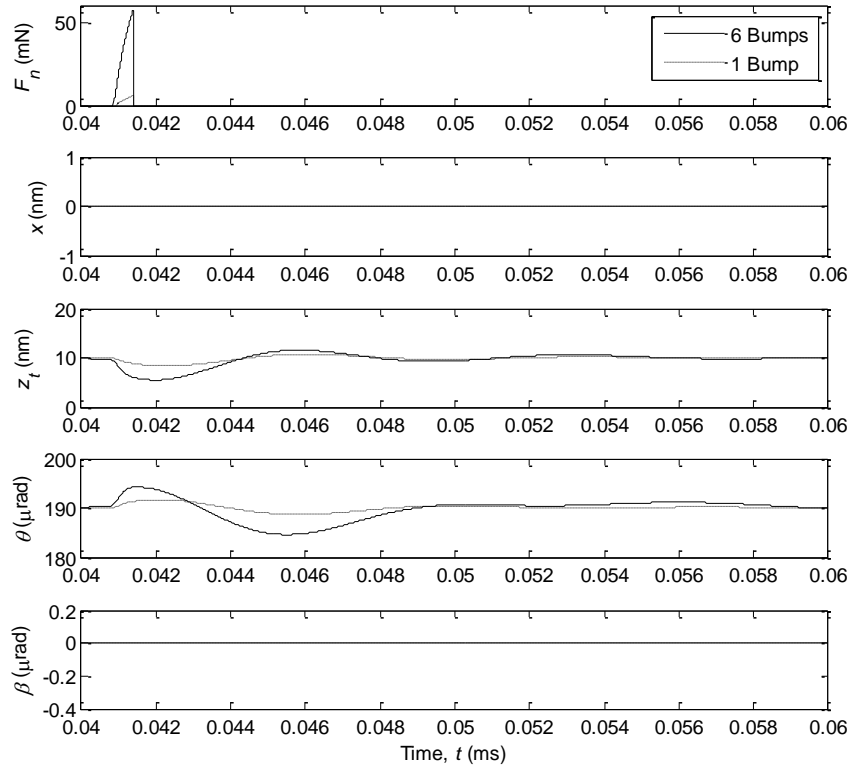

Figure 4.12 Response of the slider with Intermolecular forces to multiple bumps for bump height $h_{b}=12.5 \mathrm{~nm}$, bump radius $r_{b}=15 \mu \mathrm{m}$ and skew angle $\phi=0$ under $\beta$ decoupled conditions. 


\section{Flat Top Bump Responses}

To predict the response of the slider in the extreme conditions we assume a flat bump which has a bump radius $r_{b}=5 \mu \mathrm{m}$ and bump height $h_{b}=18 \mathrm{~nm}$ under the $10^{\circ}$ skew with intermolecular forces. We see that the flat top bump shows increased intermolecular transverse displacements and normal contact forces when compared to a paraboloidal bump because the effective area of contact (see figure 4.6) will be maximum when the bump is flat. The figure 4.13 shows the response of the slider to flat bump.
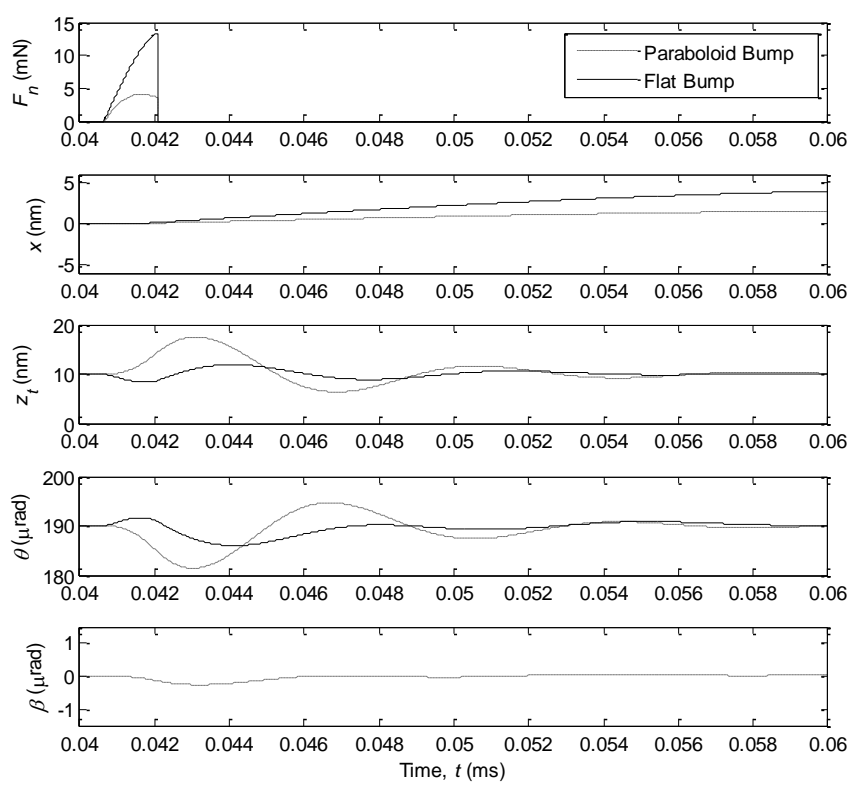

Figure 4.13 Response of the slider for a flat top bump with bump radius $r_{b}=5 \mu \mathrm{m}$, bump height $h_{b}=18 \mathrm{~nm}$ and skew angle $\phi=10^{\circ}$ under the influence of intermolecular forces.

Furthermore, the above response is compared to the response of the slider with a flat top bump under condition without intermolecular forces. The responses show that the flat top bump does not change the responses of the slider, when compared to a condition 
with a paraboloidal bump. But the response is weaker when compared to the condition with intermolecular forces as shown in the figure 4.14.
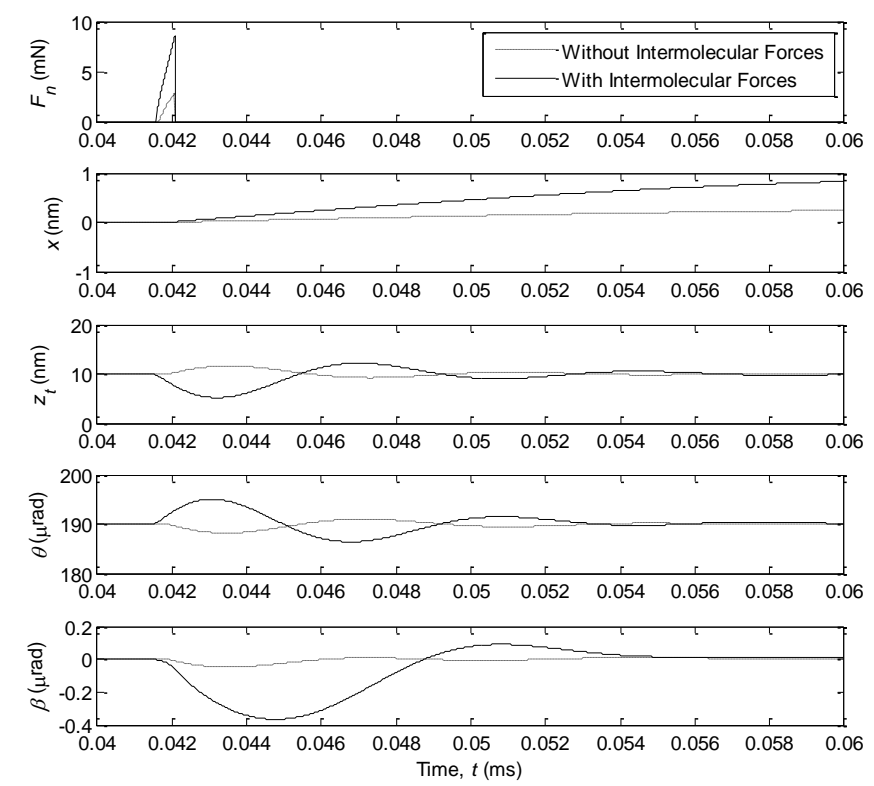

Figure 4.14 Comparison of cases for a flat top bump with and without intermolecular forces

\section{Thermally Expanding Bump Study}

Simulations were conducted for a protrusion with an equivalent base radius $r_{b}=15$ $\mu \mathrm{m}$ and at $37 \mu \mathrm{m}$ from the trailing edge, expanding towards a steady-state height $h_{b}=22$ $\mathrm{nm}$. The equivalency of $r_{b}$ for the idealized paraboloidal protrusion is based on the same resultant of the intermolecular forces as that for an actual protrusion. The figure 4.15 shows the response of the slider to the thermally actuated bump. 

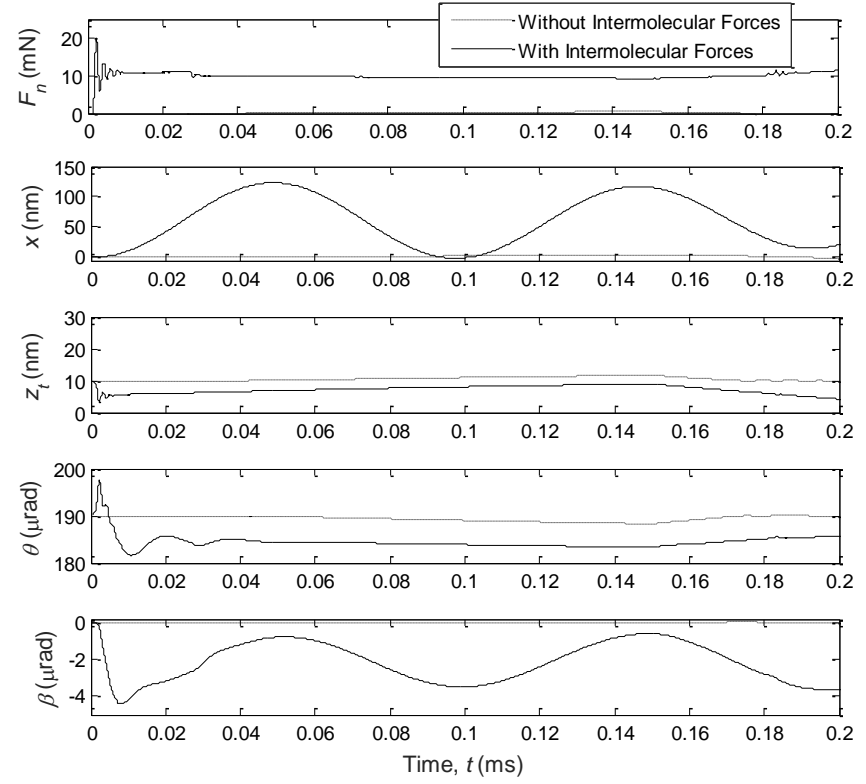

Figure 4.15 Response of the slider to the expansion and contraction of a thermally actuated protrusion for $h_{b s}=22 \mathrm{~nm}$ and skew angle $\phi=10^{\circ}$

The heating current is turned on at the time $\mathrm{t}=-0.25 \mathrm{~ms}$, and is turned off at 0.15 ms. Based on the (repulsive) normal contact force, $F_{n}$, the slider starts to contact the disk much earlier as compared to the results for a situation without intermolecular forces. After the time off slider-disk separation $\left(F_{n}\right.$ changes from a value slightly above zero to zero) for the situation without intermolecular forces, the case with intermolecular forces still shows that contact is maintained; the longer contact duration with the transverse component of the friction force causes a larger off-track displacement $\left(x_{\max }=123.1 \mathrm{~nm}\right)$ than without intermolecular forces $(2.75 \mathrm{~nm})$. Based on the trailing-edge flying height, $z_{t}$, if the contact has not broken up yet, the slider can be pulled down significantly by the intermolecular forces while the protrusion contracts due to cooling $(\mathrm{t}>0.15 \mathrm{~ms})$. 


\section{CHAPTER V}

\section{CONCLUSIONS}

A computationally efficient five-degree-of-freedom dynamic model was developed to simulate the motion of a magnetic head slider under the conditions of moving-bump collision and of contact due to an expanding protrusion on the slider for thermal flyingheight control, with consideration of intermolecular (interatomic) forces. An integrated formulation for the Lennard-Jones potential including effects of the van der Waals forces was used to characterize the intermolecular forces between the magnetic disk and the magnetic head slider. The numerical results obtained in this study shed light in the understandings of the precise behavior of the slider before, at, and after the contact with a bump or a thermal protrusion. From the analysis, the following conclusions can be drawn:

1. When a bump on the rotating disk collides with the slider, the intermolecular forces can draw the slider toward it, causing a greater normal contact force, a larger roll angle and a larger off-track displacement under nonzero skew.

2. As the bump radius increases, the maximum transverse displacement and the maximum normal contact force increase for the cases with the intermolecular forces considered, but this effect is negligible for cases without considering the intermolecular forces. 
3. When the bump height is increased, the cases without intermolecular forces show increases in the bump maximum transverse displacement and the maximum normal contact force; such increases are even larger for cases with intermolecular forces. However, the maximum normal contact force of the slider for the cases with intermolecular forces increases to a maximum and, then, decreases due to a transition from an intermolecular force-dominated regime to a collision-dominated regime.

4. The maximum transverse displacement is a linear function of the skew angle and coefficient of friction.

5. When the thermally expanding protrusion reaches a location close to the disk surface, the intermolecular forces tend to pull it into contact, resulting in an earlier contact time.

6. When the electric current on the heating element is maintained, the heating effect increases the contact force through further thermal expansion. Afterwards, when the electric heating current is turned off, the cooling process causes the protrusion to contract, but the intermolecular forces keep it in contact with the disk for a longer time before the contact breaks up, as compared to results obtained without considering the intermolecular forces. The longer contact duration with friction under nonzero skew results in a larger off-track displacement.

Some recommendations for future work would be:

1. Implement JKR model into the current program, with consideration of surface roughness effects.

2. Vary the flying height to see its effects on the slider dynamics. 
3. The bump shape can be modelled more accurately.

4. Expand the model by including the finite element analysis (FEA) of the thermally expanding stationary bump or protrusion including the determination of the time constants for thermal expansion.

The present work was submitted to the ASME/ISPS conference held on June $20-21$, 2016, and the paper was selected for publication [39]. 


\section{REFERENCES}

[1] Ono, K., Yamane, M., \& Yamaura, H. (2005). Experimental and Analytical Study of Bouncing Vibrations of a Flying Head Slider in a Near-Contact Regime. J. Tribol. Journal of Tribology, 127(2), 376. doi:10.1115/1.1843165

[2] Yoneoka, S., Owe, T., Aruga, K., Yamada, T., \& Takahashi, M. (1989). Dynamics of inline flying-head assemblies. International Magnetics Conference. doi:10.1109/intmag.1989.690146

[3] Ono, K., Yamane, M., \& Yamaura, H. (2005). Experimental and Analytical Study of Bouncing Vibrations of a Flying Head Slider in a Near-Contact Regime. J. Tribol. Journal of Tribology, 127(2), 376. doi:10.1115/1.1843165

[4] Iida, K., Ono, K., \& Yamane, M. (2002). Dynamic Characteristics and Design Consideration of a Tripad Slider in the Near-Contact Regime. J. Tribol. Journal of Tribology, 124(3), 600. doi:10.1115/1.1467089

[5] Ono, K., \& Takahashi, K. (1999). Analysis of Bouncing Vibrations of a 2-DOF Tripad Contact Slider Model With Air Bearing Pads Over a Harmonic Wavy Disk Surface. J. Tribol. Journal of Tribology, 121(4), 939. doi:10.1115/1.2834159

[6] Wang, R., Nayak, V., Huang, F., Tang, W., \& Lee, F. (2001). Head-Disk Dynamics in the Flying, Near Contact, and Contact Regimes. J. Tribol. Journal of Tribology, 123(3), 561. doi:10.1115/1.1308037

[7] Cha, E., \& Bogy, D. B. (1995). Numerical Simulations of Slider Interaction With Multiple Asperity Using Hertzian Contact Model. J. Tribol. Journal of Tribology, 117(4), 575. doi:10.1115/1.2831518

[8] Ono, K., \& Nakagawa, K. (2005). Experimental Study of Bouncing Behavior of Spherical Sliders on Stationary Magnetic Disks. World Tribology Congress III, Volume 1. doi:10.1115/wtc2005-63628 
[9] Kohira, H., Tanaka, H., Matsumoto, M., \& Talke, F. E. (2001). Investigation of Slider Vibrations Due to Contact With a Smooth Disk Surface. J. Tribol. Journal of Tribology, 123(3), 616. doi:10.1115/1.1308045

[10] Chen, D., \& Bogy, D. B. (2010). Numerical Investigation of Bouncing Vibrations of an Air Bearing Slider in Near or Partial Contact. J. Tribol. Journal of Tribology, 132(1), 011901. doi:10.1115/1.4000514

[11] Yuan, Y., \& Wang, S. (2009). A Simplified Dynamic Model for the Analysis of the Slider Off-Track Motion Due to Head-Disk Interactions. J. Tribol. Journal of Tribology, 131(2), 021902. doi:10.1115/1.3063808

[12] Wu, L., \& Bogy, D. B. (2002). Effect of the Intermolecular Forces on the Flying Attitude of Sub-5 NM Flying Height Air Bearing Sliders in Hard Disk Drives. J. Tribol. Journal of Tribology, 124(3), 562. doi:10.1115/1.1456454

[13] Li, J., Liu, B., Hua, W., \& Ma, Y. (2002). Effects of intermolecular forces on deep sub-10 nm spaced sliders. IEEE Trans. Magn. IEEE Transactions on Magnetics, 38(5), 2141-2143. doi:10.1109/tmag.2002.802690

[14] Thornton, B., \& Bogy, D. (2003). Head-disk interface dynamic instability due to intermolecular forces. IEEE Trans. Magn. IEEE Transactions on Magnetics, 39(5), 24202422. doi:10.1109/tmag.2003.816424

[15] Thornton, B., \& Bogy, D. (2004). A Parametric Study of Head-Disk Interface Instability Due to Intermolecular Forces. IEEE Trans. Magn. IEEE Transactions on Magnetics, 40(1), 337-344. doi:10.1109/tmag.2003.821156

[16] Deoras, S. K., \& Talke, F. E. (2007). Effect of Intermolecular Forces on the Dynamic Response of a Slider. J. Tribol. Journal of Tribology, 129(1), 177. doi:10.1115/1.2390720

[17] Kurita, M., Shiramatsu, T., Miyake, K., Kato, A., Soga, M., Tanaka, H., Suk, M. (2006). Active flying-height control slider using MEMS thermal actuator. Microsystem Technologies Microsyst Technol, 12(4), 369-375. doi:10.1007/s00542-006-0104-4

[18] Wang, R., Wu, X., Weresin, W., \& Ju, Y. S. (2001). Head protrusion and its implications on head-disk interface reliability. IEEE Trans. Magn. IEEE Transactions on Magnetics, 37(4), 1842-1844. doi:10.1109/20.950985 
[19] Ju, Y. (2005). Self-heating in thin-film magnetic recording heads due to write currents. IEEE Trans. Magn. IEEE Transactions on Magnetics, 41(12), 4443-4448. doi:10.1109/tmag.2005.858697

[20] Nikitin, V., Gider, S., Tabib, J., Hsiao, D., Salo, M., Sui, G., . . Maruyama, Y. (2004). Spatial and Temporal Profiling of Protrusion in Magnetic Recording Heads. IEEE Trans. Magn. IEEE Transactions on Magnetics, 40(1), 326-331. doi:10.1109/tmag.2003.821159

[21] Xu, J., Kurita, M., \& Tokuyama, M. (2004). Thermal Analysis of a Magnetic Head. IEEE Trans. Magn. IEEE Transactions on Magnetics, 40(4), 3142-3144. doi:10.1109/tmag.2004.830208

[22] Aoki, K., Hoshino, T., Iwase, T., Imamura, T., \& Aruga, K. (2005). Thermal pole-tip protrusion analysis of magnetic heads for hard disk drives. IEEE Trans. Magn. IEEE Transactions on Magnetics, 41(10), 3043-3045. doi:10.1109/tmag.2005.855253

[23] Pust, L., Rea, C., \& Gangopadhyay, S. (2002). Thermomechanical head performance. IEEE Trans. Magn. IEEE Transactions on Magnetics, 38(1), 101-106. doi:10.1109/tmag.2002.988919

[24] Juang, J., \& Bogy, D. B. (2007). Air-Bearing Effects on Actuated Thermal Pole-Tip Protrusion for Hard Disk Drives. J. Tribol. Journal of Tribology, 129(3), 570. doi:10.1115/1.2736456

[25] Davis, T. A., \& Sigmon, K. (2005). MATLAB primer. Boca Raton, FL: Chapman \& Hall/CRC.

[26] Adamson, A. W., \& Gast, A. P. (1997). Physical chemistry of surfaces. New York: Wiley.

[27] Johnson, K. L. (1985). Contact mechanics. Cambridge: Cambridge University Press.

[28] Suh, A. Y., \& Polycarpou, A. A. (2005). Adhesive contact modeling for sub-5-nm ultralow flying magnetic storage head-disk interfaces including roughness effects. J. Appl. Phys. Journal of Applied Physics, 97(10), 104328. doi:10.1063/1.1914951

[29] Shi, X., \& Zhao, Y. (2004). Comparison of various adhesion contact theories and the influence of dimensionless load parameter. Journal of Adhesion Science and Technology, 18(1), 55-68. doi:10.1163/156856104322747009 
[30] Greenwood, J. A. (1997). Adhesion of elastic spheres. Proceedings of the Royal Society A: Mathematical, Physical and Engineering Sciences, 453(1961), 1277-1297. doi:10.1098/rspa.1997.0070

[31] Johnson, K., \& Greenwood, J. (1997). An Adhesion Map for the Contact of Elastic Spheres. Journal of Colloid and Interface Science, 192(2), 326-333. doi: $10.1006 /$ jcis. 1997.4984

[32] Derjaguin, B., Muller, V., \& Toporov, Y. (1975). Effect of contact deformations on the adhesion of particles. Journal of Colloid and Interface Science, 53(2), 314-326. doi:10.1016/0021-9797(75)90018-1

[33] Tabor, D. (1977). Surface Forces and Surface Interactions. Plenary and Invited Lectures, 3-14. doi:10.1016/b978-0-12-404501-9.50009-2

[34] Davis, T. A., \& Sigmon, K. (2005). MATLAB primer. Boca Raton, FL: Chapman \& Hall/CRC

[35] Adamson, A. W., \& Gast, A. P. (1997). Physical chemistry of surfaces. New York: Wiley.

[36] Tabor, D. (1977). Surface forces and surface interactions. Journal of Colloid and Interface Science, 58(1), 2-13. doi:10.1016/0021-9797(77)90366-6

[37] Israelachvili, J. N. (1992). Intermolecular and surface forces. London: Academic Press.

[38] Lim, C. (2007). A Study of the Behavior of the Hard-Disk Interface in Computer Hard Disk Drives. School of Mechanical and Aerospace engineering-Nanyang Technological University.

[39] Pathak, S., and Wang, S., 2016, “A Dynamic Model of the Magnetic Head Slider with Contact and Off-Track Motion Due to a Thermally Actuated Protrusion or a Moving Bump Involving Intermolecular Forces," Proceedings of the 2016 ASME Information Storage and Processing Systems Conference, Santa Clara, California, June 20, 2016, Paper Number ISPS2016-9614, pp. 1-3. 
[40] Binnig, G., Quate, C. F., \& Gerber, C. (1993). Atomic Force Microscope. Scanning Tunneling Microscopy Perspectives in Condensed Matter Physics, 55-58. doi:10.1007/978-94-011-1812-5_4

[41] Binnig, G., Gerber, C., Stoll, E., Albrecht, T., \& Quate, C. (1987). Atomic resolution with atomic force microscope. Surface Science, 189-190, 1-6. doi:10.1016/s00396028(87)80407-7

[42] Atomic Force Microscopy: Revolutionizing the Future of Nano-scale Imaging. (2009, November 21). DUJS Online. Retrieved from http://dujs.dartmouth.edu/2009/11/atomicforce-microscopy-revolutionizing-the-future-of-nano-scale-imaging/\#.V31zz7grKM9 
APPENDICES 
APPENDIX A

\section{LIST OF SYMBOLS}

$a=$ bump radius, $\mathrm{m}$

$\boldsymbol{C}=$ damping matrix of the 5DOF system

$c=$ damping matrix of the air bearing

$c_{\text {arm }}=$ damping coefficient of the actuator arm, $\mathrm{N} \mathrm{s} / \mathrm{m}$

$c_{d}=$ disk damping coefficient, $\mathrm{N} \mathrm{s} / \mathrm{m}$

$D=$ vertical distance from the origin of the slider coordinate system to that of the disk

coordinate system, $\mathrm{m}$

$\boldsymbol{F}=$ force vector, $\mathrm{N}$

$F_{i}=$ intermolecular forces

$F_{n}=$ normal contact force, $\mathrm{N}$

$F_{n, \text { ave }}=$ average contact force, $\mathrm{N}$

$F_{n, \text { max }}=$ maximum contact force, $\mathrm{N}$

$F_{t}=$ transverse component of the friction force, $\mathrm{N}$

$H=$ slider thickness, $\mathrm{m}$ 
$h_{b}=$ bump height, $\mathrm{m}$

$I_{\mathrm{arm}}=$ moment of inertia of the arm, $\mathrm{kg} \mathrm{m} 2$

$I_{\theta}=$ roll moment of inertia, $\mathrm{kg} \mathrm{m} 2$

$I_{\beta}=$ pitch moment of inertia, $\mathrm{kg} \mathrm{m} 2$

$\boldsymbol{K}=$ suspension stiffness matrix

$\boldsymbol{K}_{c}=$ combined system stiffness matrix

$\boldsymbol{k}=$ air bearing stiffness matrix

$k_{\text {arm }}=$ actuator arm stiffness, $\mathrm{N} / \mathrm{m}$

$k_{d}=$ disk stiffness, $\mathrm{N} / \mathrm{m}$

$L=$ slider length, $\mathrm{m}$

$\boldsymbol{L}=$ vector from the center of the bump base to an arbitrary point on the bottom surface of the slider, $\mathrm{m}$

$l=$ length of the rear pad, $\mathrm{m}$

$\boldsymbol{M}=$ mass matrix

$M_{\theta}=$ roll moment, $\mathrm{N} \mathrm{m}$

$M_{\beta}$ pitch moment, $\mathrm{N} \mathrm{m}$

$m=$ slider mass, $\mathrm{kg}$

$m_{\text {arm }}$ mass of the actuator arm, $\mathrm{kg}$

$n=$ rotational speed of the disk, rpm 
$\boldsymbol{n}=$ inward unit normal vector of the slider bottom

$r=$ distance from the center of mass of the slider to the rotational axis of the arm, $\mathrm{m}$

$R_{c}=$ contact time ratio

$T=$ period of the contact force, $\mathrm{s}$

$\boldsymbol{T}=$ vector from the origin to the tangential point, $\mathrm{m}$

$t_{c}=$ contact time of a single cycle, $\mathrm{s}$

$\tau=$ time constants

$\boldsymbol{X}=$ displacement vector, $\mathrm{m}$

$x=$ transverse displacement of the slider, $\mathrm{m}$

$x_{\text {arm }}=$ transverse displacement of the actuator arm, $\mathrm{m}$

$x_{b}=x$-coordinate of the bump summit, $\mathrm{m}$

$x_{c}=x$-coordinate of the contact point, $\mathrm{m}$

$x_{p, \max }=$ maximum $x$-coordinate of the rear pad corners, $\mathrm{m}$

$x_{p, \min }=$ minimum $x$-coordinate of the rear pad corners, $\mathrm{m}$

$y_{b}=y$-coordinate of the bump summit, $\mathrm{m}$

$y_{c}=y$-coordinate of the contact point, $\mathrm{m}$

$z=$ vertical displacement of the center of mass of the slider, $\mathrm{m}$

$z_{t}=$ flying height at the center of the trailing edge, $\mathrm{m}$ 
$z_{t 0}=$ flying height at the center of the trailing edge in the equilibrium state, $\mathrm{m}$

$\beta=$ roll angle, rad

$\delta=$ offset of the bump path from the center line of the slider, $\mathrm{m}$

$\Delta_{n}=$ interference between the slider and bump, $\mathrm{m}$

$\theta=$ pitch angle, $\mathrm{rad}$

$\theta_{0}=$ pitch angle in the equilibrium state, $\mathrm{rad}$

$\lambda=$ bump spacing, $\mathrm{m}$

$\mu=$ coefficient of friction

$\xi=$ shape factor of the contact force

$\phi=$ skew angle, $\mathrm{rad}$ 


\section{APPENDIX B}

\section{PARABOLOIDAL BUMP FORMULATION}

The generalized parabola equation is given as

$$
z=A y^{2}+B y+c
$$

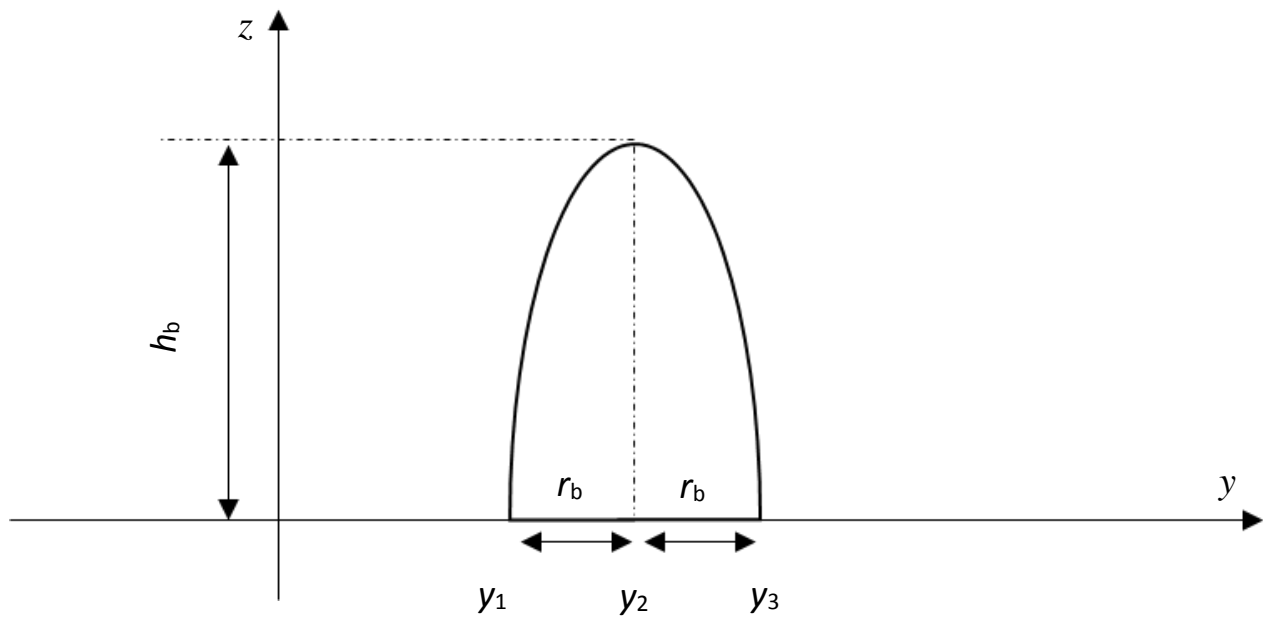

Figure B.1 The formulation for a paraboloidal bump

From the above figure, we have three points on the $\mathrm{y}$-axis defined as the $y_{1}\left(\mathrm{y}_{1}, 0\right), y_{2}\left(\mathrm{y}_{2}, h_{\mathrm{b}}\right)$, and $y_{3}\left(y_{3}, 0\right)$, where $h_{\mathrm{b}}$ is the height of the bump and $r_{\mathrm{b}}$ is the radius of the bump. 
At $y_{1}$,

$$
\begin{gathered}
0=A y_{1}{ }^{2}+B y_{1}+c \\
-c=A y_{1}{ }^{2}+B y_{1}
\end{gathered}
$$

Eqn B-2

Eqn B-3

At $y_{2}$,

$$
h_{b}=A y_{2}{ }^{2}+B y_{2}+c
$$

Eqn B-4

At $y_{3}$

$$
h_{b}=A y_{3}^{2}+B y_{3}+c
$$

Eqn B-5

Writing $r_{\mathrm{b}}$ in terms of $y_{1}, y_{2}$ and $y_{3}$

$$
\begin{aligned}
& y_{1}=y_{2}-r_{b} \\
& y_{3}=y_{2}+r_{b}
\end{aligned}
$$

Eqn B-7

Then the equations B-3, and B-5 become,

$$
\begin{aligned}
& A\left(y_{2}-r b\right)^{2}+B\left(y_{2}-r_{b}\right)+c=0 \\
& A\left(y_{2}+r b\right)^{2}+B\left(y_{2}+r_{b}\right)+c=0
\end{aligned}
$$

Eqn B-8

Eqn B-9 
Solving the equations B-8 and B-9, we get

$$
\begin{array}{cc}
A\left(\left(y_{2}-r_{b}\right)^{2}-\left(y_{2}+r_{b}\right)^{2}\right)+B\left(\left(y_{2}-r_{b}\right)-\left(y_{2}+r_{b}\right)\right)=0 & \text { Eqn B-10 } \\
A\left(4 r b y_{2}\right)+B\left(2 r_{b}\right)=0 & \text { Eqn B-11 } \\
B=-2 y_{2} A & \text { Eqn B-12 }
\end{array}
$$

Substitute the equation B-12 into equation B-8 and we get

$$
\begin{array}{cc}
A\left(y_{2}-r_{b}\right)^{2}-2 y_{2} A\left(y_{2}-r_{b}\right)+c=0 & \text { Eqn B-13 } \\
A\left(y_{2}{ }^{2}+r b^{2}-2 y_{2} r b\right)^{2}-2 y_{2} A\left(y_{2}-r_{b}\right)+c=0 & \text { Eqn B-14 } \\
A\left(r b^{2}-y_{2}{ }^{2}\right)^{2}+c=0 & \text { Eqn B-15 } \\
c=-A\left(r b^{2}-y_{2}{ }^{2}\right)^{2} & \text { Eqn B-16 }
\end{array}
$$

Substituting B-16 and B-12 in B-4

$$
\begin{gathered}
A+\left(-2 y_{2} A\right) y_{2}+-A\left(r_{b}{ }^{2}-y_{2}{ }^{2}\right)^{2}=h_{b} \\
-A r b^{2}=h_{b} \\
A=-\frac{h_{b}}{r_{b}^{2}}
\end{gathered}
$$

Eqn B-18

Eqn B-19 
Substituting B-19 in B-16 and B-12, we get

$$
\begin{gathered}
B=-2 y_{2}\left(\frac{-h_{b}}{r_{b}{ }^{2}}\right) \\
B=\frac{2 y_{2} h_{b}}{r_{b}{ }^{2}} \\
c=-\left(-\frac{h_{b}}{r_{b}{ }^{2}}\right)\left(r_{b}{ }^{2}-y_{2}{ }^{2}\right)^{2} \\
c=h_{b}-\frac{y_{2}^{2}}{r_{b}{ }^{2}}
\end{gathered}
$$

Eqn B-20

Eqn B-21

Eqn B-22

Eqn B-23

Hence, the equation of a Paraboloidal Bump is given as

$$
\left(-\frac{h_{b}}{r_{b}{ }^{2}}\right) y^{2}+\left(\frac{2 y_{2} h_{b}}{r_{b}{ }^{2}}\right) y+h_{b}-\frac{y_{2}{ }^{2}}{r_{b}{ }^{2}}=z
$$




\section{APPENDIX C HAMAKER CONSTANT CALCULATION}

The equation used for calculating the Intermolecular forces between the slider and the disk was calculated using the equation 3-7, from the chapter III. The terms $h$ in the equation denotes the distance between the slider and disk, $A$ is the Hamaker constant, $B$ is a constant. The terms A and B were calculated with respect to the material properties of the slider and the disk in the given procedure [12].

$$
A=\pi^{2} \times C \times \rho_{1} \times \rho_{2} \quad \text { Eqn C-1 }
$$

And

$$
B=\pi^{2} \times D \times \rho_{1} \times \rho_{2} \quad \text { Eqn C-2 }
$$

where $C, D$ are constants, $\rho_{1}$ is the number density of the platter (disk), $\rho_{2}$ is the number density of the slider. $C=10^{-17} \mathrm{Jm}^{6}$ and $D=10^{-134} \mathrm{Jm}^{12}$ [16,37]. For the platter, $\rho_{1}=$ $6.027 \times 10^{28} \mathrm{~m}^{-3}$ and for the disk, $\rho_{2}=1.18 \times 10^{29} \mathrm{~m}^{-3}[38]$. So $A$ and $B$ when calculated were found to be $7.02 \times 10^{-19} \mathrm{~J}$ and $7.02 \times 10^{-76} \mathrm{Jm}^{6}$. 


\section{APPENDIX D}

\section{RAW DATA FIGURES FOR COMPARISONS}

The below given are the supporting raw figures for the case of $F_{\mathrm{n}}$ vs $r_{\mathrm{b}}$
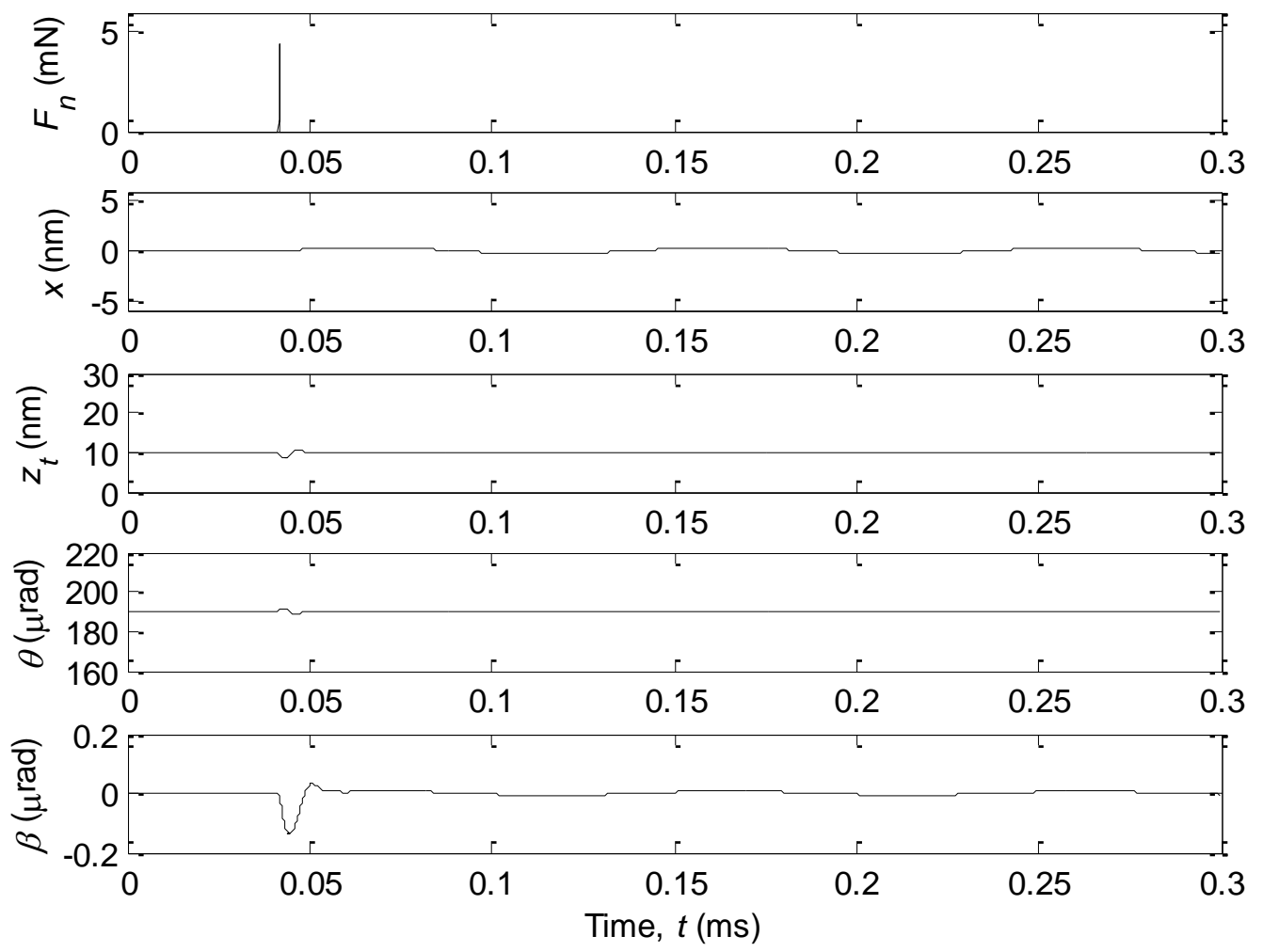

Figure D.1 The response graphs for bump height $h_{\mathrm{b}}=18 \mathrm{~nm}$, radius of bump $r_{\mathrm{b}}=12 \mu \mathrm{m}$ and skew $\phi=10^{\circ}$ 

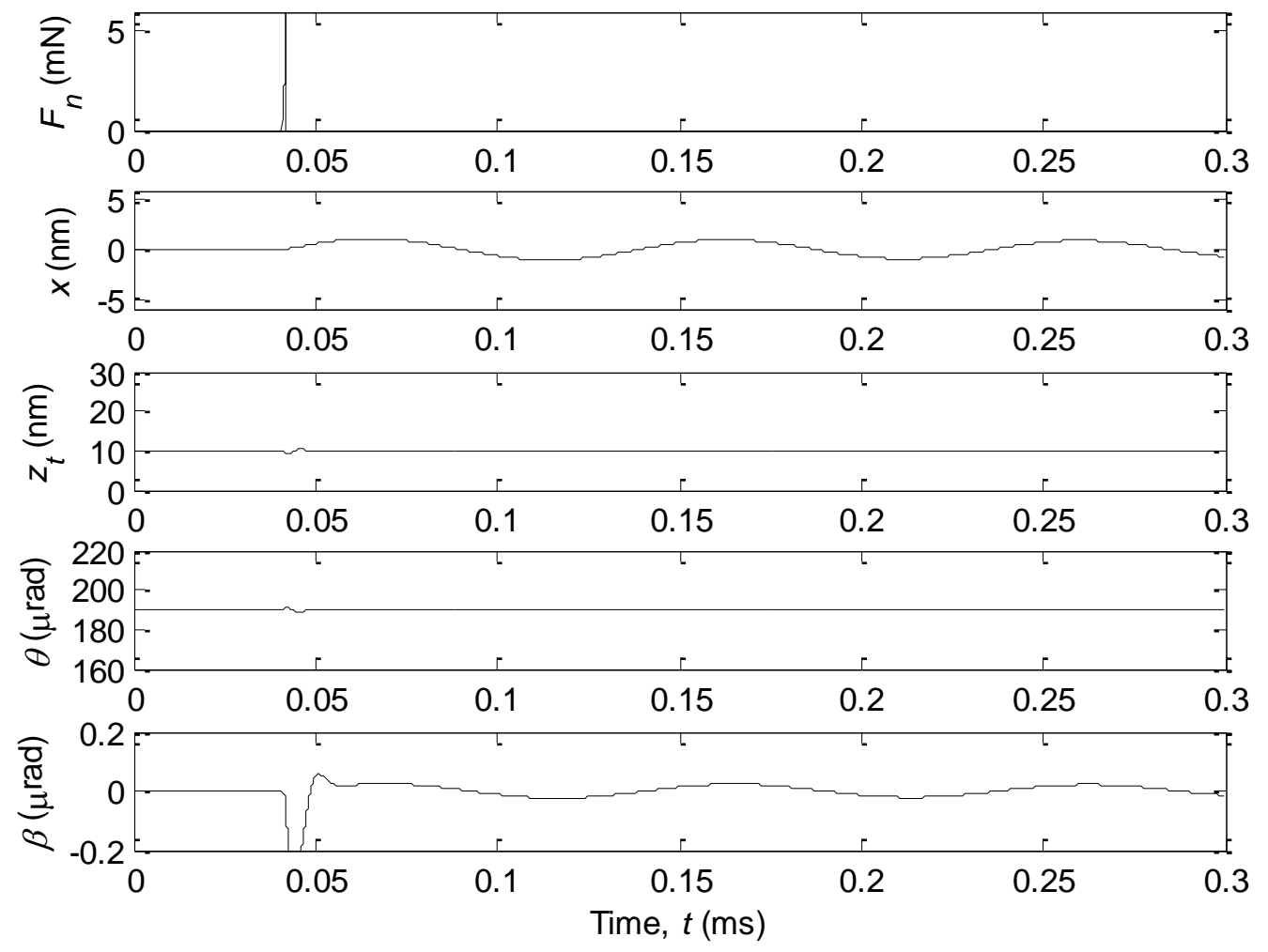

Figure D.2 The response graphs for bump height $h_{\mathrm{b}}=18 \mathrm{~nm}$, radius of bump $r_{\mathrm{b}}=14 \mu \mathrm{m}$ and skew $\phi=10^{\circ}$ 

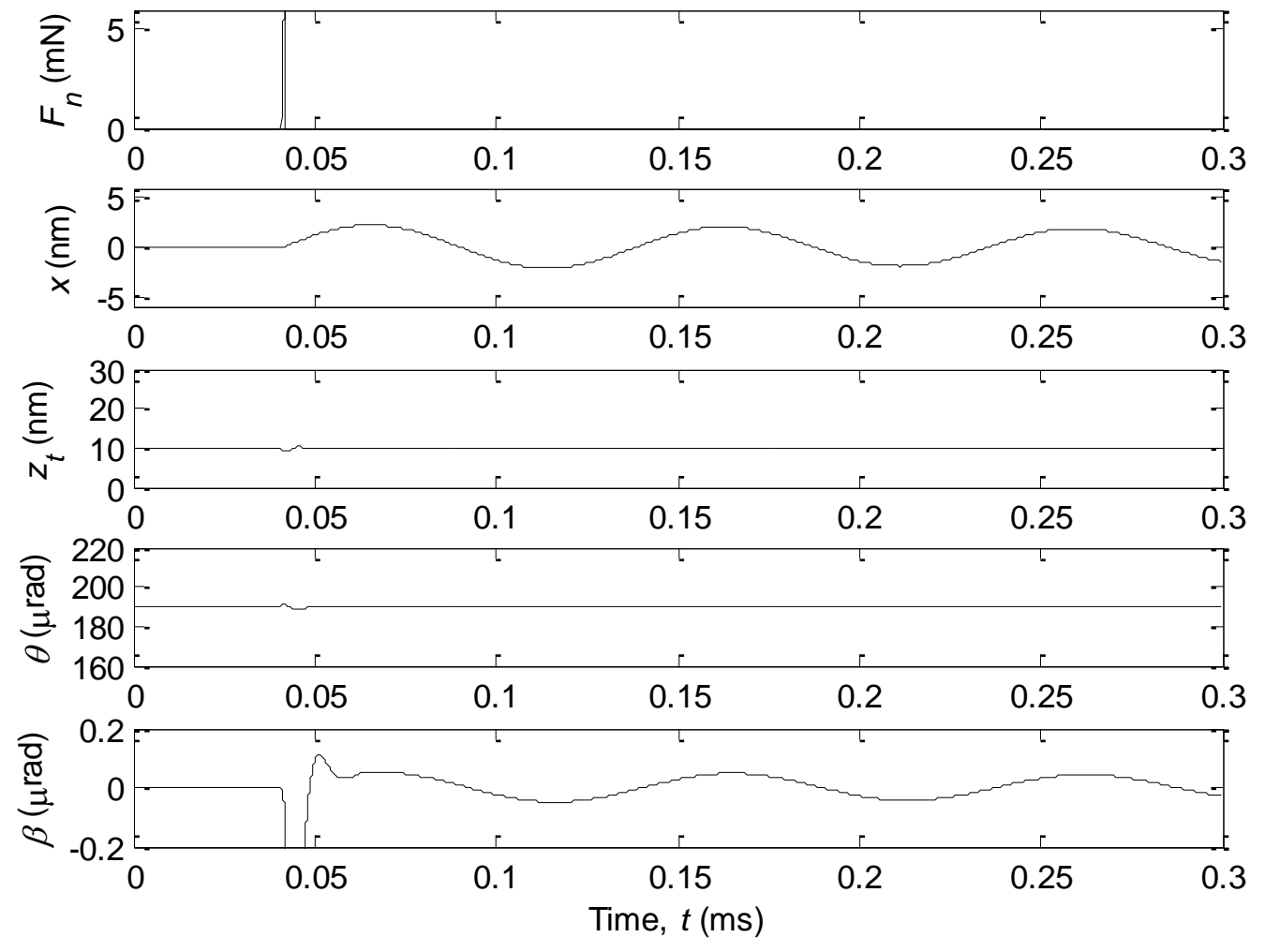

Figure D.3 The response graphs for bump height $h_{\mathrm{b}}=18 \mathrm{~nm}$, radius of bump $r_{\mathrm{b}}=16 \mu \mathrm{m}$ and skew $\phi=10^{\circ}$ 

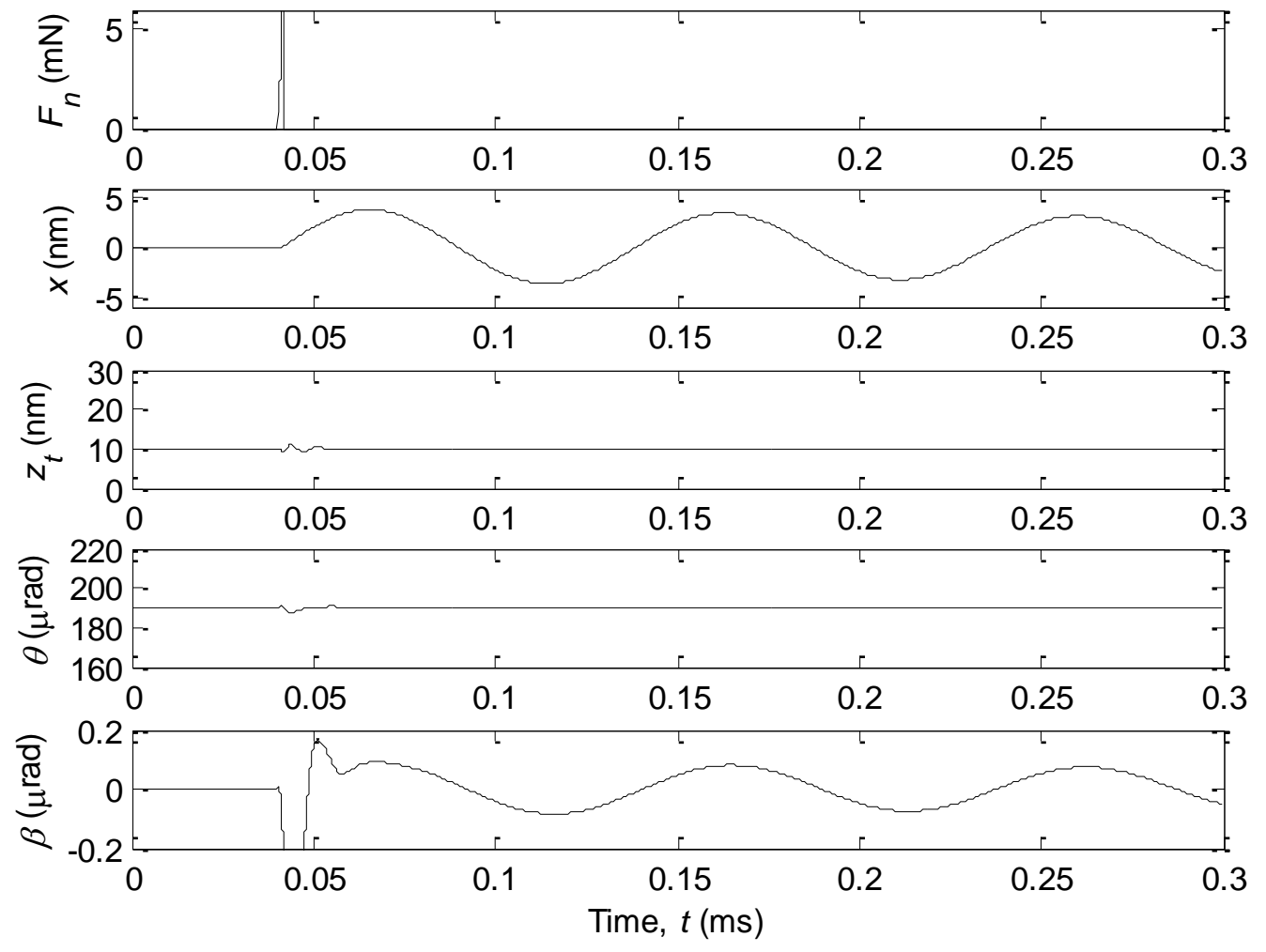

Figure D.4 The response graphs for bump height $h_{\mathrm{b}}=18 \mathrm{~nm}$, radius of bump $r_{\mathrm{b}}=18 \mu \mathrm{m}$ and skew $\phi=10^{\circ}$ 

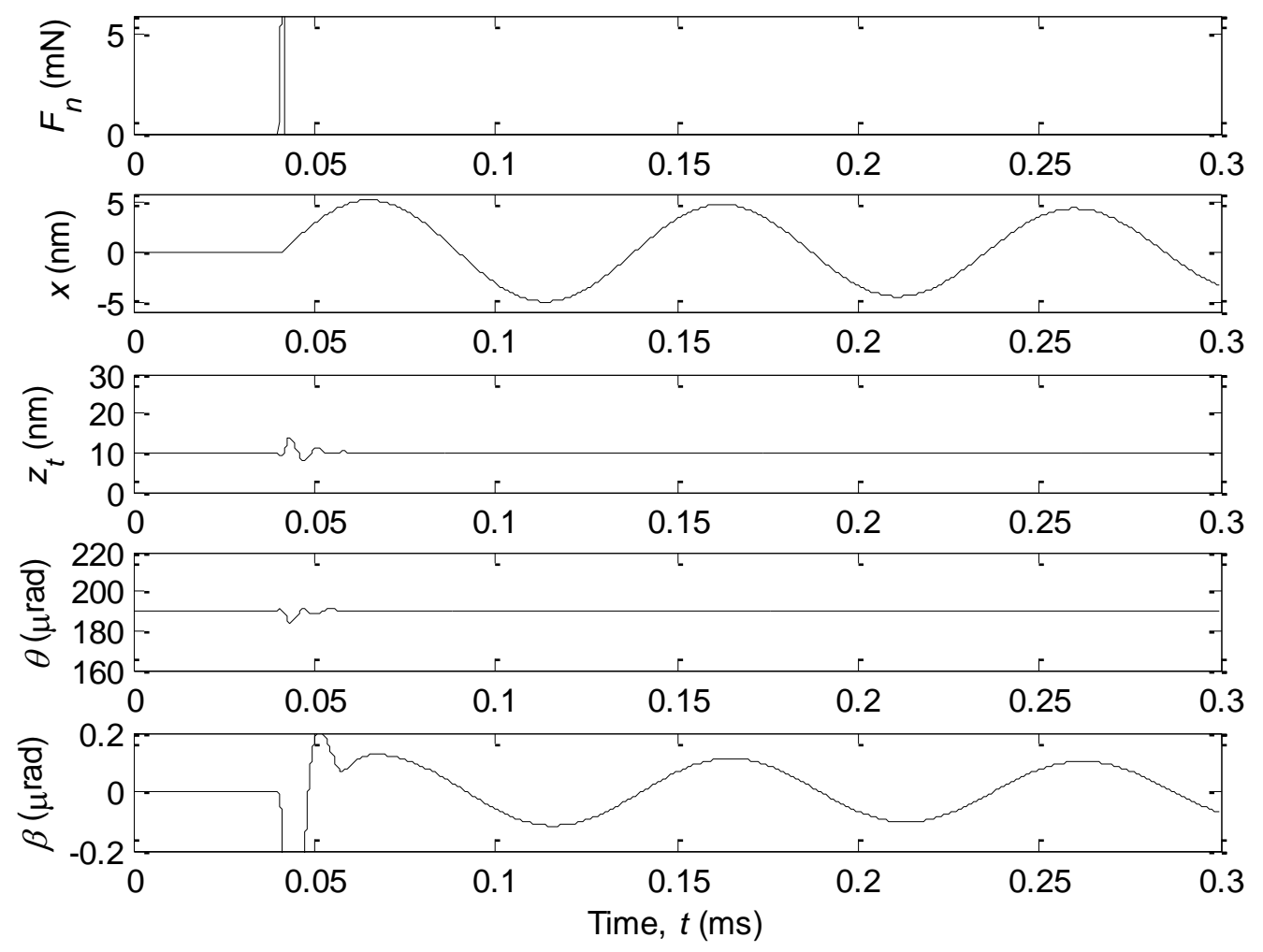

Figure D.5 The response graphs for bump height $h_{\mathrm{b}}=18 \mathrm{~nm}$, radius of bump $r_{\mathrm{b}}=20 \mu \mathrm{m}$ and skew $\phi=10^{\circ}$ 

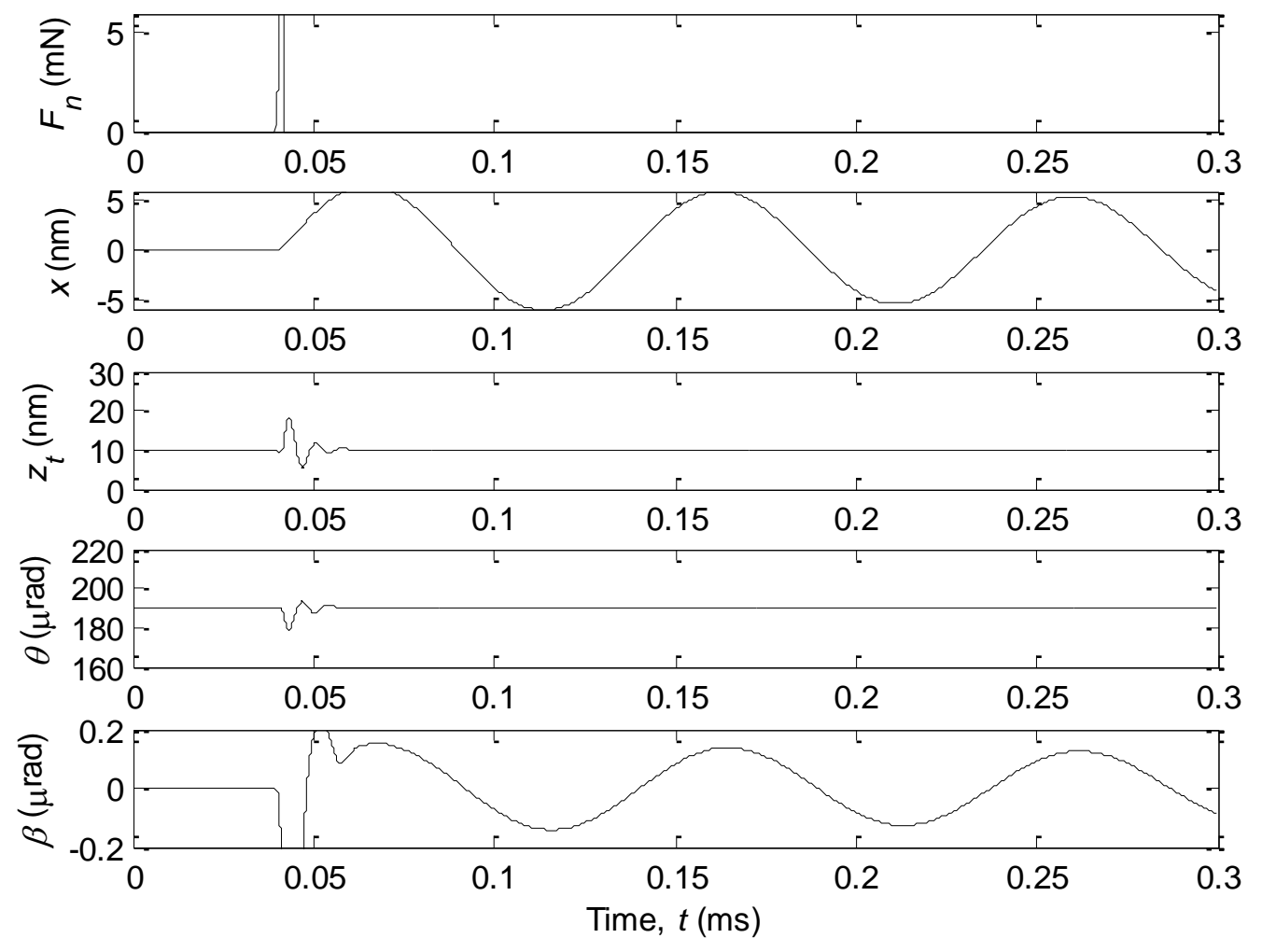

Figure D.6 The response graphs for bump height $h_{\mathrm{b}}=18 \mathrm{~nm}$, radius of bump $r_{\mathrm{b}}=22 \mu \mathrm{m}$ and skew $\phi=10^{\circ}$ 

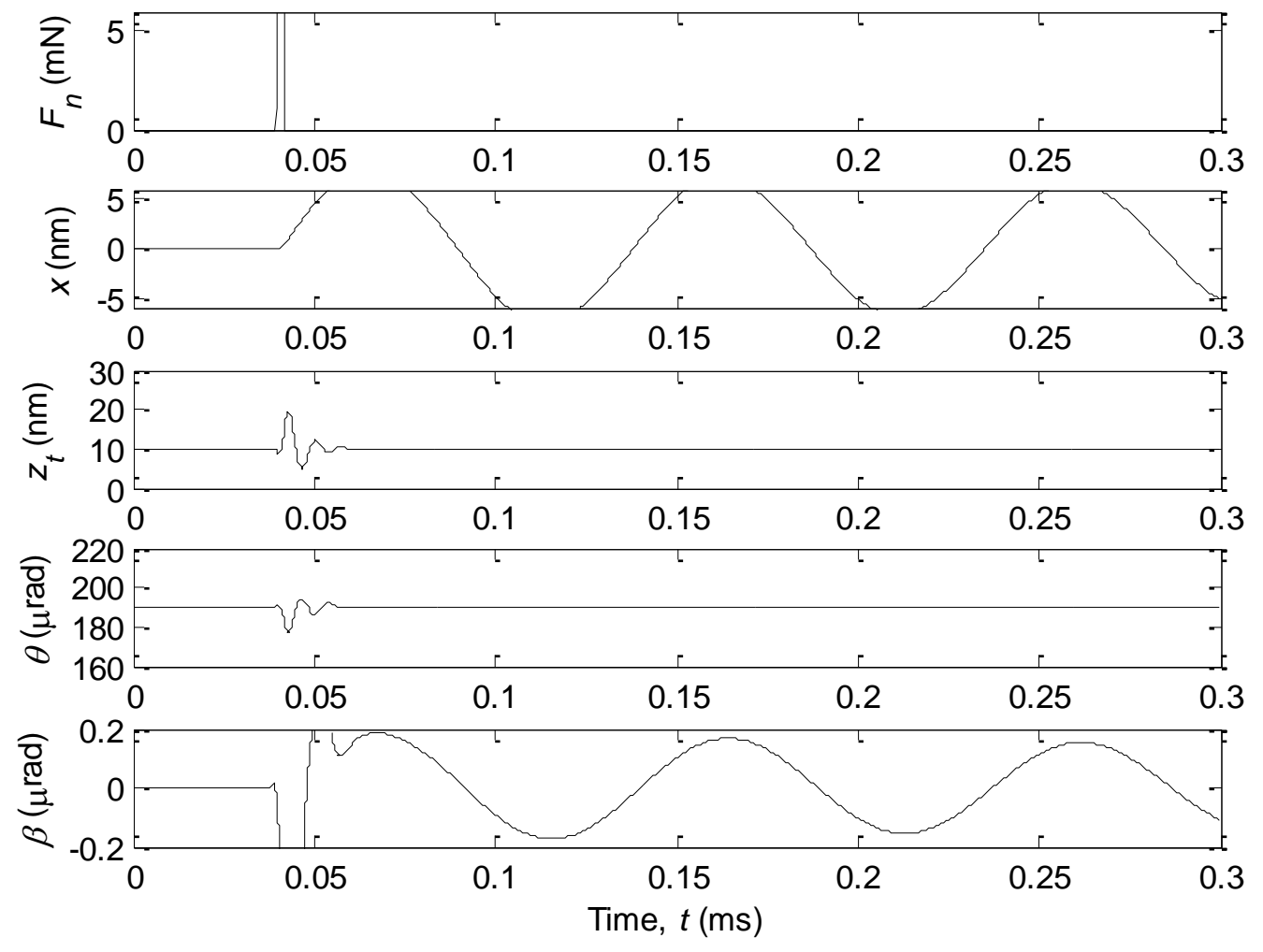

Figure D.7 The response graphs for bump height $h_{\mathrm{b}}=18 \mathrm{~nm}$, radius of bump $r_{\mathrm{b}}=24 \mu \mathrm{m}$ and skew $\phi=10^{\circ}$ 

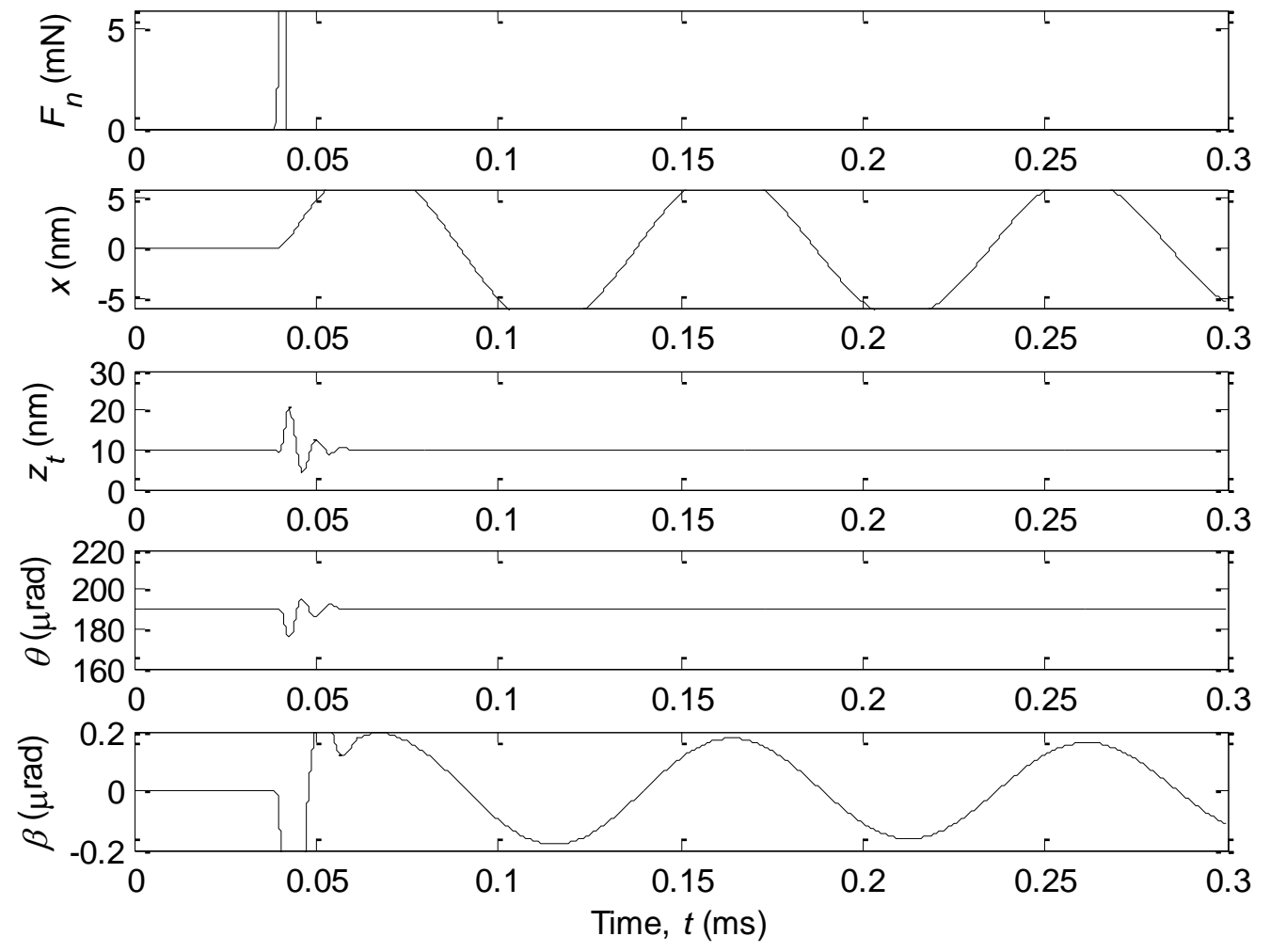

Figure D.8 The response graphs for bump height $h_{\mathrm{b}}=18 \mathrm{~nm}$, radius of bump $r_{\mathrm{b}}=26 \mu \mathrm{m}$ and skew $\phi=10^{\circ}$ 

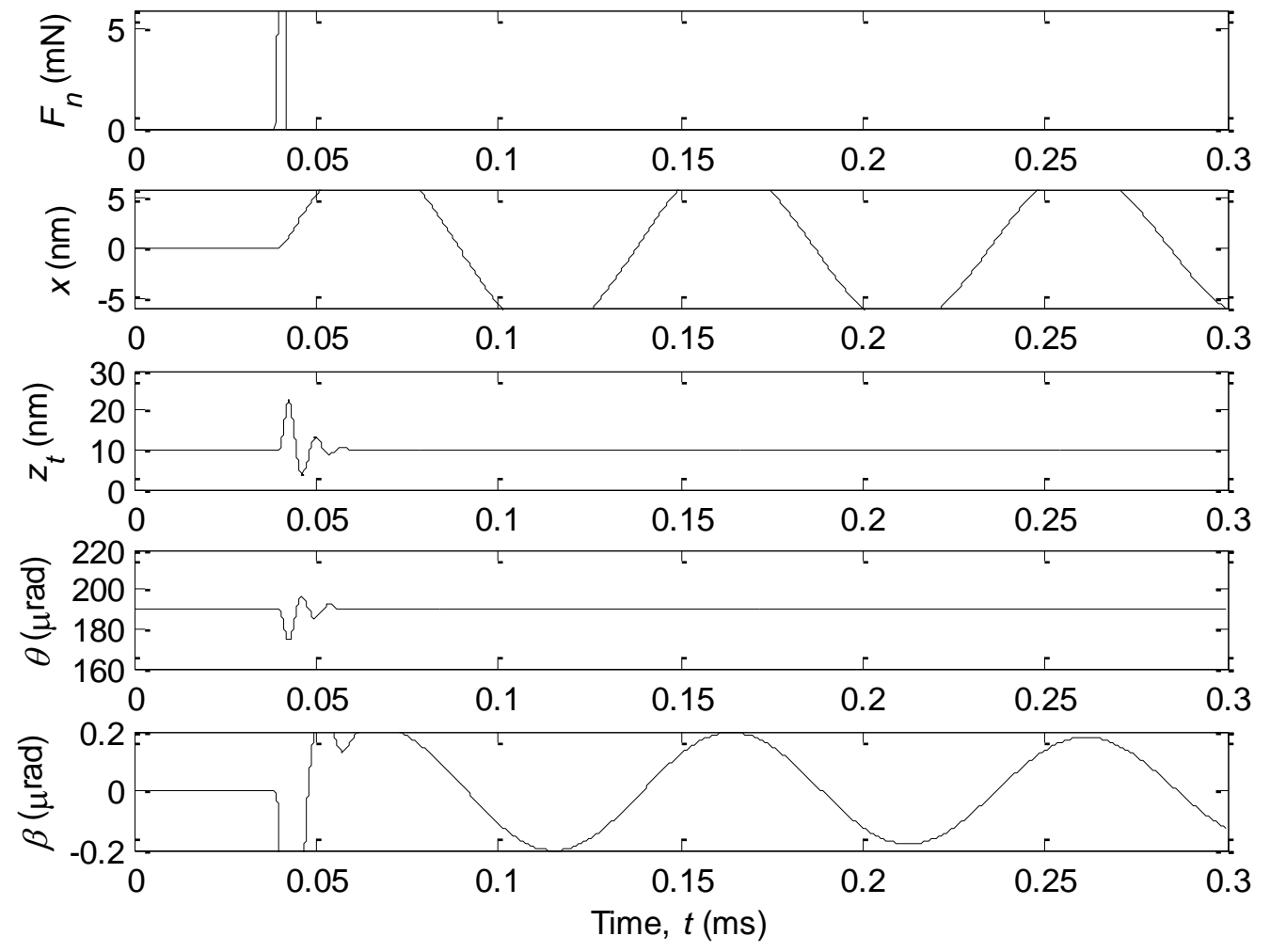

Figure D.9 The response graphs for bump height $h_{\mathrm{b}}=18 \mathrm{~nm}$, radius of bump $r_{\mathrm{b}}=28 \mu \mathrm{m}$ and skew $\phi=10^{\circ}$ 

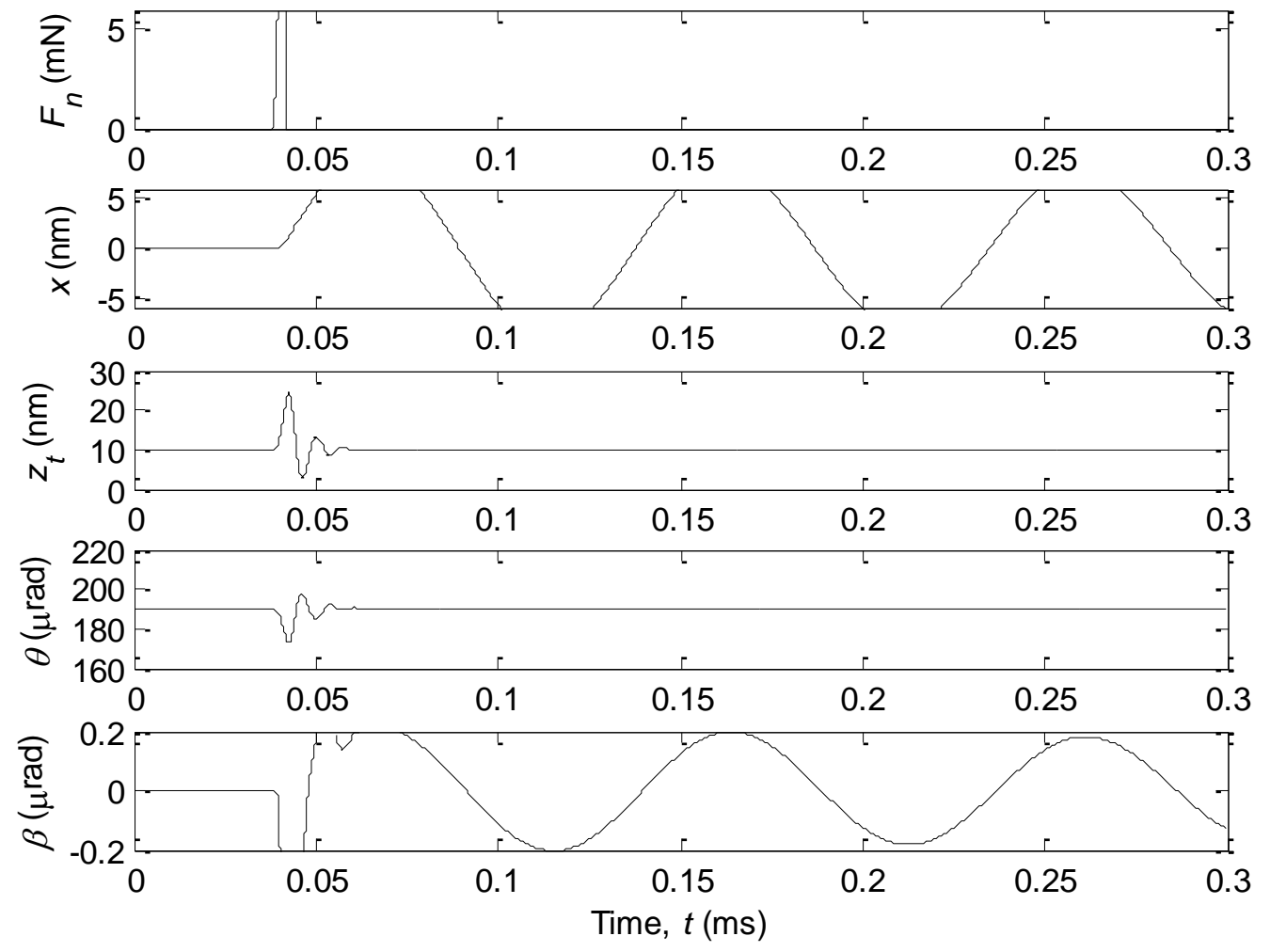

Figure D.10 The response graphs for bump height $h_{\mathrm{b}}=18 \mathrm{~nm}$, radius of bump $r_{\mathrm{b}}=30 \mu \mathrm{m}$ and skew $\phi=10^{\circ}$ 


\section{APPENDIX E}

\section{CALLING TREE'S FOR THE MATLAB PROGRAM}

The following flowchart shows the calling pattern of the MATLAB program.

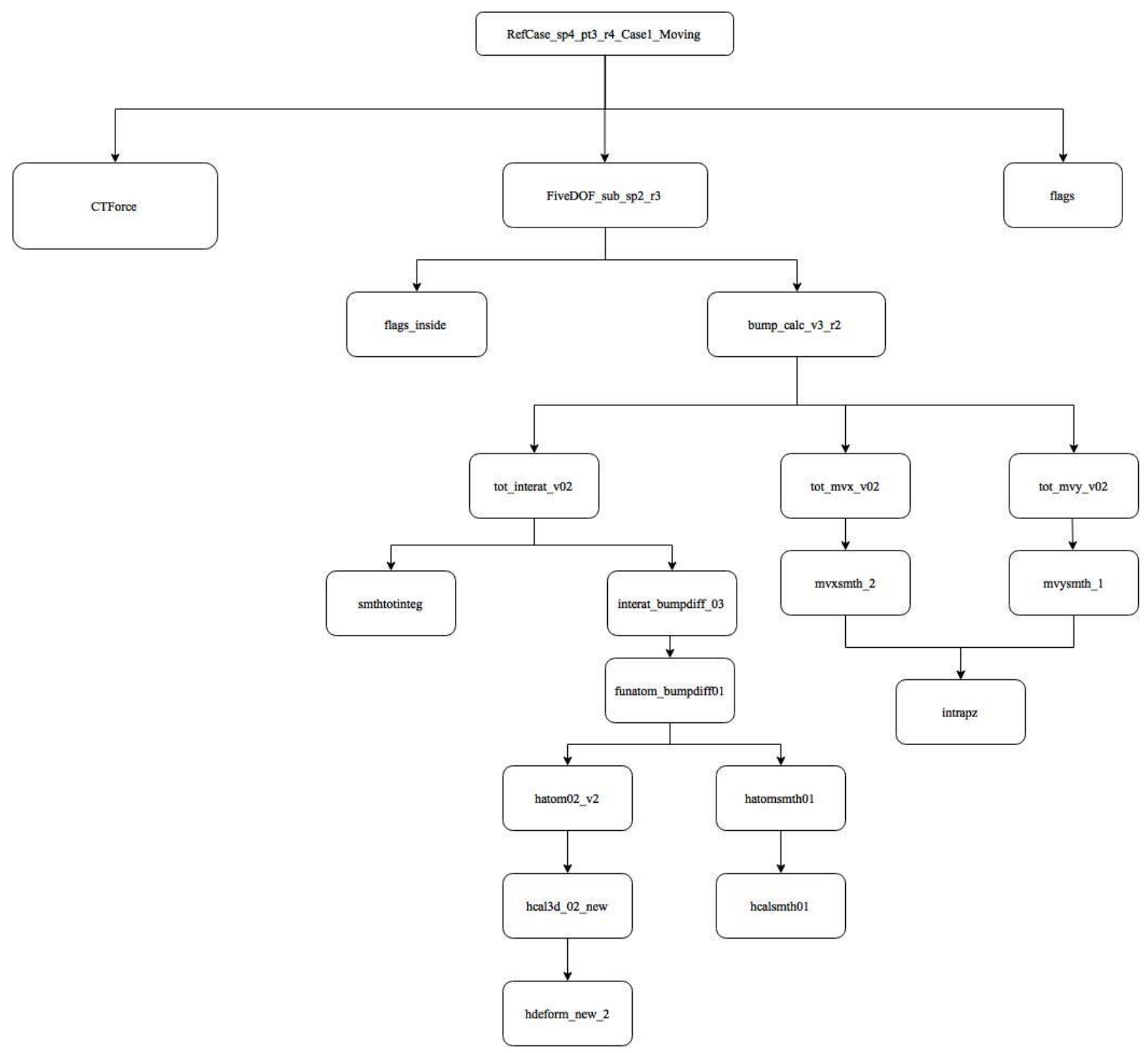

